PALEO

Revue d'archéologie préhistorique

$17 \mid 2005$

Varia

\title{
Réflexions méthodologiques sur les études de matières premières lithiques
}

1 - Des lithothèques au matériel archéologique

Methodological considerations about lithic studies. 1 - from lithotheques to

archaeological samples

Alain Turq

\section{OpenEdition}

\section{Journals}

Édition électronique

URL : http://journals.openedition.org/paleo/883

DOI : 10.4000/paleo.883

ISSN : 2101-0420

Éditeur

SAMRA

Édition imprimée

Date de publication : 1 décembre 2005

Pagination : 111-132

ISSN : 1145-3370

\section{Référence électronique}

Alain Turq, «Réflexions méthodologiques sur les études de matières premières lithiques », PALEO [En ligne], 17 | 2005, mis en ligne le 23 avril 2010, consulté le 07 juillet 2020. URL : http:// journals.openedition.org/paleo/883; DOI : https://doi.org/10.4000/paleo.883

Ce document a été généré automatiquement le 7 juillet 2020

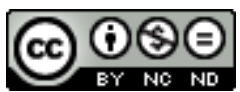

PALEO est mis à disposition selon les termes de la licence Creative Commons Attribution - Pas d'Utilisation Commerciale - Pas de Modification 4.0 International. 


\title{
Réflexions méthodologiques sur les études de matières premières lithiques
}

\author{
1 - Des lithothèques au matériel archéologique \\ Methodological considerations about lithic studies. 1 - from lithotheques to \\ archaeological samples
}

Alain Turq

1 L'origine des matériaux utilisés par les hommes préhistoriques intéresse, depuis longtemps, les archéologues (Lartet et Christy 1869 ; Combes 1888 ; Boule 1892 ; SaintPerrier 1930). A partir des années cinquante elle sera une préoccupation récurrente (Coulonges et Sonneville-Bordes 1953; Bordes et Sonneville-Bordes 1954 ; Monméjean et al. 1964 ; Valensi 1960 ; Lorblanchet 1964 ; Lumley Brandi 1969; Fitte 1970 ; Demars 1974 ; Bricker 1975; Seronie-Vivien et Le Tensorer 1977; Turq 1977a, 1977b; Le Tensorer 1979).

2 Dans les années quatre-vingt les bases méthodologiques sont posées et l'étude lithologique devient un véritable sujet de recherche (Morala 1979, 1980,1983, 1989 ; Demars 1980 ; Duchadeau-Kervazo 1982 ; Rigaud 1982 ; Larrick 1983; Chadelle 1983 ; Geneste 1985 ; Seronie-Vivien 1987 ; Le Brun Ricalens 1988) ${ }^{1}$.

3 A partir des années quatre-vingt-dix, le succès de l'approche est tel que pratiquement toutes les études traitant d'un ensemble lithique sont accompagnées d'une étude de l'origine des matériaux utilisés.

4 Devant l'importance prise par les études lithologiques et les résultats qu'elles gênèrent, il nous est apparu aujourd'hui nécessaire de mener une réflexion sur les limites et la fiabilité de l'outil de travail, domaine qui a fait l'objet de peu de travaux (Geneste 1985, Seronie Vivien 1987, Turq 1992, 2003). Ce travail, qui se veut de portée générale, repose sur une longue expérience acquise dans l'approvisionnement en matières premières lithiques des chasseurs-cueilleurs (depuis le début du Paléolithique initial jusqu'à la fin du Mésolithique) principalement du Nord-Est aquitain. Dans cette première partie ${ }^{2}$, 
nous traiterons des travaux de terrain (cartographie, estimation des gites), de la caractérisation des échantillons bruts issus des affleurements actuels, des outils ainsi constitués que sont les lithothèques, des difficultés de passer de l'évaluation des ressources actuelles à l'estimation des ressources passées et des échantillons de références aux objets archéologiques.

\section{1 - Caractérisation des matériaux}

5 Elle a comme objectif de définir les caractères propres des matériaux puis, de les classer par groupes plus ou moins homogènes voire spécifiques.

\section{1 - Les méthodes de caractérisation}

6 Au début, deux approches se sont opposées : celle des pratriciens de terrain (Demars 1980, 1982a et 1982b; Morala 1980 ; Torti 1983) et celle des analystes (Masson 1981 et 1982). Chacune avait ses avantages et ses inconvénients. Les premiers, avec une démarche rapide et empirique, se sont contentés d'une approche macroscopique pour identifier les types de silex régionaux. Souvent, cette méthode n'a pas permis de reconnaître les matériaux extérieurs à la zone étudiée. Les seconds (analystes, micropaléontologues, pétrographes), insistaient sur une caractérisation avant tout géologique. Cette méthode plus scientifique, mais aussi plus coûteuse en temps, nécessitant parfois des interventions lourdes (lames minces, analyses), n'aurait pu s'avérer efficace que si elle avait reposé sur une bonne connaissance du terrain. Un consensus s'est mis rapidement en place. L'étude macroscopique avec l'aide d'une loupe binoculaire, la prise en compte des données micropaléontologiques et de la structure de la silicification se sont avérées performantes (Mauger 1985). Aujourd'hui, la majeure partie des chercheurs a adopté la proposition faite par M. et M.-R. Séronie-Vivien (1987) pour la description de la structure du silex et l'emploi du langage utilisé pour la description des microfaciès carbonatés (Dunham 1962), bien adapté à la pétrotexture de ces matériaux (Bonvin-Borer, Masserrey 1981). Le type d'organisation des éléments constitutifs, la distinction entre la matrice (fond) et les éléments figurés (grains) correspondant aux fossiles, minéraux et pellets permettent de définir plusieurs types de structures: packstone, wackestone, mudstone, grainstone (fig. 1). De plus, la généralisation de l'analyse micropaléontologique par examen macroscopique ainsi que le recours plus systématique à des lames minces permettent la détermination des microorganismes, bioclastes, l'observation des micro-structures de la roche (souvent caractéristiques), la présence d'hydroxydes, de minéraux opaques, de fantômes de minéraux ou de restes recristallisés. L'examen au microscope électronique à balayage (M.E.B) donne accès à la nanostructure de la roche et apporte des renseignements sur le contenu siliceux de l'échantillon et sur les types de silice représentés. Par exemple, quatre types morphologiques de silice ont été décrits dans les silex du sud-est de la France (Rio et Chalamet 1980). Ces différentes formes de silice et leur quantification dans un échantillon s'avèrent être, dans certains cas, de bons éléments distinctifs entre plusieurs silex (Grégoire 2000 et 2001). 
Figure 1 - Principaux types de microfaciés des silex (d'après Seronie-Vivien M. et M.-R. 1987) Figure 1 - Principal types of microfacies of flint (after Seronie-Vivien M. and M.-R. 1987).
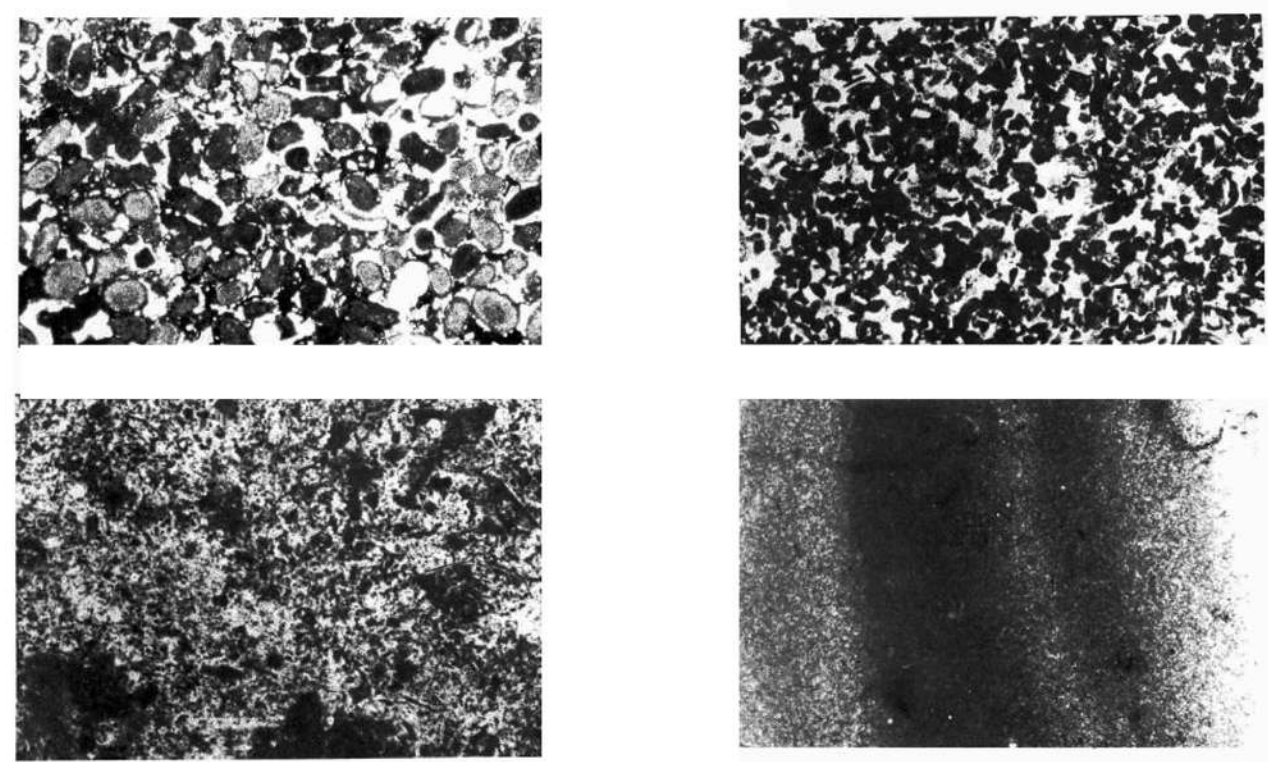

A, Texture grainstone, SVX. 38 -X 35. Jaspe jaune moutarde, Chantrezac (Charente) Lias inférieur (Sinémurien) (cliché du bas) (pl. 5) ; B, Texture packstone, SVX. 201 - X 17. Silex du Bergeracois, Lanquais (Dordogne), Maestrichien (pl. 35) ; C, Texture wackestone, SVX. 177 - X 20. Silex blond du Sénonien, Saint-Félix-de-Reillac-et-Mortemart (Dordogne), Campanien (pl. 31) ; D, Texture mudstone, SVX. 197 - X 18. Silex du Fumélois, Duravel (Lot), Turonien (pl. 23).

A, Texture grainstone, SVX. 38 -X 35. Jasper yellow, Chantrezac (Charente) Lower Lias (Sinemurian) (bottom view) (pl. 5) ; B, Texture packstone, SVX. 201 - X 17. Bergerac flint, Lanquais (Dordogne), Maestrichian (pl. 35) ; C, Texture wackestone, SVX. 177 - X 20. beige senonian flint, Saint-Félix-de-Reillac-et-Mortemart (Dordogne), Campanian (pl. 31) ; D, Texture mudstone, SVX. 197 - X 18. Fumel flint, Duravel (Lot), Turonian (pl. 23).

7 Plusieurs types de caractérisations physico-chimiques sont également à notre disposition: Activation Neutronique (Horan 1977 ; Luedtke 1978, 1979) ; Particle-Induced Xray Emission (Consigny 1996); Laser Ablation Inductivly Coupled Plasma Mass Spectrometry (Morisset 1996; Blet 1999); Inductivly Coupled Plasma coupled with Atomic Emission Spectrometry (Bressy 1998, 2002 ; Bressy et al. 1998, 1999). Destructives ou non, elles permettent de détecter les éléments chimiques présents dont la répartition quantitative est directement induite par le milieu et les conditions de formation de la roche siliceuse (Rio 1982). Bien qu'ayant donné ponctuellement des résultats, elles présentent deux inconvénients majeurs : leur coût qui réduit le nombre d'échantillons pouvant être traités et le faible taux de résultats positifs. Tous les objets ne pouvant être analysés, l'essentiel de la détermination repose essentiellement sur une comparaison macroscopique entre les échantillons analysés et le reste des pièces archéologiques.

\section{2 - Une typologie}

8 L'indispensable mise en place d'une typologie s'est d'abord faite de manière individuelle avant que les discussions de la réunion des Eyzies aient autorisé les premières corrélations entre les appellations proposées par chacun des chercheurs et permis de jeter les bases d'un vocabulaire vernaculaire commun publié par J.-M. Geneste (1985). Ainsi, la classe 3 de P.-Y. Demars (1980), le type 1 de R. Larrick (1983), 
M.P.1 de J.-P. Chadelle (1983), le type X de A. Morala (1980) s'appellent désormais « silex gris et noir du Sénonien ».

9 La classification préconisée est calquée sur le cheminement de l'analyse. Les pièces sont d'abord attribuées à des groupes larges que nous qualifions de génériques : silex gris ou noirs du Sénonien, meulières et silex lacustre... et silex des altérites. Ce dernier groupe se définit par les modifications physico-chimiques qui ont profondément modifié leur structure (zonation, recristallisation, encroûtements ferralitiques) et qui, très souvent, ont amélioré considérablement leur qualité mécanique (Bergeracois, GrandPressigny...). La poursuite de l'analyse permet d'attribuer une partie d'entre eux à des types plus précis qualifiés de marqueurs lithologiques (tabl. 1) alors caractéristique de zones géographiques plus ou moins précises en raison de leurs spécificités pétrographiques (silex à pyrite du Sarladais) et par leur contenu paléontologique (silex de Belvès). En ce qui concerne le vocabulaire, la préférence a été donnée aux termes vernaculaires, «silex du Fumélois» ou du «Bergeracois». Ce système présente l'avantage d'être compris par tous les chercheurs au moins régionaux et ne nécessite pas un retour à la publication princeps pour savoir quel type se cache derrière un code. En outre ce principe facilite la prise en compte de l'évolution de nos connaissances sans bousculer la nomenclature existante.

Tableau 1 - Classification des types de silex du nord-est du Bassin aquitain en groupes peu spécifiques et types particuliers ou marqueurs lithologiques.

Table 1 - Classification of flint types from North east Aquitain Basin in groups: less specific ones and specific types or lithological markers.

\begin{tabular}{|l|l|}
\hline Groupes génériques & Types spécifiques ou marqueurs lithologiques \\
\hline $\begin{array}{l}\text { Silex gris et noirs du } \\
\text { Sénonien }\end{array}$ & $\begin{array}{l}\text { - silex de Belvès (Campanien inférieur à Subalveolina Dordonica major) } \\
\text { - silex à pyrite du Sarladais }\end{array}$ \\
\hline Silex beige du Sénonien & $\begin{array}{l}\text { - silex de Belvès (Campanien inférieur à Subalveolina Dordonica major) } \\
\text { - silex de Gavaudun (Coniacien du Haut-Agenais) } \\
\text { - silex à Lepidorbitoides sp. des formations sénoniennes du sud de la } \\
\text { Garonne }\end{array}$ \\
\hline Meulières et silex lacustres & Silex lacustres tertiaires de la bordure du Massif central \\
\hline Silex zonés du Sénonien & Silex du Bergeracois à Orbitoïdes media \\
\hline Silex jaspoïdes & Silex jaspoïde de l'Hettangien (oolithes) \\
\hline Silex du Turonien & Silex du Fumélois \\
\hline
\end{tabular}

\section{3 - Principales difficultés d'identification}

10 Les principales difficultés concernent la rareté des éléments caractéristiques et les altérations subies par les objets archéologiques qu'elles soient d'origine naturelle ou anthropique. 


\section{Rareté des éléments caractéristiques}

11 La description pétrographique des échantillons tant naturels qu'archéologiques a pour but de mettre en évidence les éléments caractéristiques d'un étage géologique, d'une formation ou d'un faciès. Elle doit être comparée avec des modèles sédimentaires et les données paléogéographiques régionales (Seronie-Vivien 1972; Platel 1989). Très souvent l'attribution repose sur la présence d'un fossile particulier. Dans ce cas, même si cet organisme est abondant dans la formation géologique, sa présence est aléatoire dans le volume des objets que nous étudions. Prenons comme exemple, orbitoides media fossile marqueur du Campanien supérieur du nord-est du Bassin aquitain. $\mathrm{Si}$, exceptionnellement, on peut observer 4 ou 5 spécimens sur $10 \mathrm{~cm}^{2}$, il n'est pas rare non plus de ne pas trouver un seul fossile sur des surfaces 20 à 30 fois plus importantes.

\section{Altérations}

12 Après leur abandon ou leur perte par l'homme préhistorique, les silex sont soumis à des modifications liées soit à l'anthropisation soit à des phénomènes naturels qui peuvent rendre difficile, voire impossible, leur identification lithologique. Nous n'évoquerons ici que deux des phénomènes les plus gênants : l'action du feu et celle de la patine. Le premier, provoque de nombreux changements de couleur et de structure qui le plus souvent interdisent toute identification (Séronie Vivien 1995). Le second a des effets révélateurs, gênants ou mutilants. Dans un premier exemple, le silex que R. Simonnet (1999) appelle grain de mil ne prend son aspect caractéristique que lorsqu'il est patiné (fig. 2-3). A l'état frais, c'est un matériau blond uniforme (fig. 2-3 en haut) ${ }^{3}$, couleur que gardera par la suite la "pâte » alors que les nombreux débris qu'il contient blanchiront (fig. 2-3 en bas). Dans un second exemple, nous avons à faire à un simple voile qui peut oblitérer en partie la structure mais aussi mettre en évidence les fossiles en raison d'une modification différentielle des composantes du silex. Enfin si l'altération est trop forte, elle peut interdire toute analyse ce qui est souvent le cas pour des séries anciennes de surface ou trouvés en stratigraphie (La Micoque) où les objets sont devenus de couleur jaune clair uniforme très poreux et légers. 
Figure 2

Figure 2

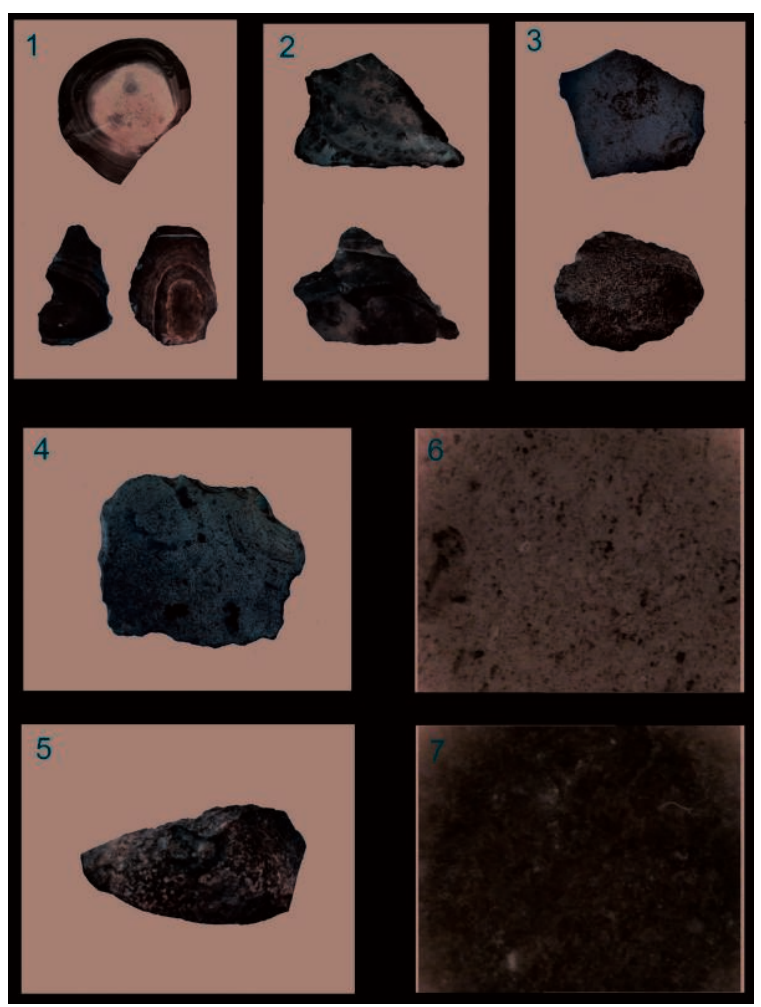

\section{Le facteur humain}

13 C'est un sujet délicat que nous ne pouvons pas passer sous silence. A partir des années quatre-vingt-dix, pour accompagner leur étude de séries archéologiques d'une approche lithologique, des étudiants ou des chercheurs ont travaillé seuls. Sans formation et parfois sans recherche bibliographique sérieuse, les résultats obtenus sont faussés, (par exemple Bongni 1994). Ces données qui risquent d'être considérées comme acquises, peuvent être reprises dans d'autres travaux. Si l'on ne dispose ni du temps ni de la volonté de se former, il convient de confier cette étude à un spécialiste ou la faire avec lui. L'idéal serait un travail d'équipe où le premier tri serait fait par une personne qui connaît bien les ressources locales et fera appel à d'autres pour confirmer son classement des matières premières apparemment allochtones ou inhabituelles et leur en confiera l'analyse.

\section{4 - Fiabilité et limites de l'approche actuelle}

14 Si l'on reprend la classification proposée (Turq 1992, 1999 et tabl. 1), pour 75 à $85 \%$ des pièces, l'étude s'arrêtera à l'attribution à une zone géographique large. La présence d'un fossile caractéristique, d'une texture et d'une organisation très particulière permet d'extraire des groupes précédents 10 à $15 \%$ des objets et de leur attribuer des caractères lithologiques et paléontologiques qui, le plus souvent, correspondent à un étage géologique. Une localisation plus précise, comme la présence d'un élément attribuable à un espace géographique restreint, est encore plus rare: exemple Subalveolina dordonica pour le Campanien inférieur du sud et centre du Périgord. 


\section{5 - Conclusion}

L'identification de l'origine des matières premières lithiques comporte un certain nombre de difficultés et d'incertitudes. Elle dépend essentiellement de la connaissance des affleurements, de leur contenu, de la maille de l'échantillonnage, de la connaissance des conditions de dépôt et de formation des accidents siliceux, de l'évolution des silicifications et du cortège micropaléontologique. L'état d'avancement des recherches ne permet encore que de percevoir, dans les grandes lignes, leur origine. Dans de telles conditions, lorsque l'on passe à l'interprétation des données, nous ne pouvons formuler que des probabilités plus ou moins fortes.

\section{2 - Les lithothèques : l'outil de base de toute étude lithologique}

16 Avant toute étude lithologique de séries archéologiques, il convient de recenser, caractériser et classer les sources de matières premières lithiques actuelles, c'est-à-dire de créer une lithothèque. Ainsi, dans le nord-est du Bassin aquitain, chaque chercheur (Valensi 1960 ; Demars 1980 ; Morala 1980 ; Rigaud 1982 ; Chadelle 1983 ; Geneste 1985 ; Séronie Vivien 1987 ; Le Brun Ricalens 1988 ; Turq 1988c, 1992 ; Célérier 1993) a, dans son travail, utilisé son propre référentiel. Ces outils d'analyse sont hétérogènes puisque, outre leur spécificité géographique, ils reflètent les préoccupations de leur auteur.

\section{1 - Bilan des pratiques actuelles}

Trois aspects peuvent être distingués: les travaux de terrain, leur représentation graphique et les fiches d'inventaire. Avant même d'aller plus loin, rappelons que tout inventaire doit prendre en considération tous les types de lieux dans lesquels les hommes préhistoriques étaient susceptibles de trouver le silex. Ces lieux communément appelés gîtes (Demars $1980:$ p. 46) correspondent à différentes étapes de leur transport, depuis la position en place dans la roche mère jusqu'aux formations alluviales ou cordons littoraux (fig. 3). 
Figure 3 - Bloc diagramme synthétique des différents types de gîtes à silex. Figure 3 - Synthetic block diagram of the different types of flint sources.

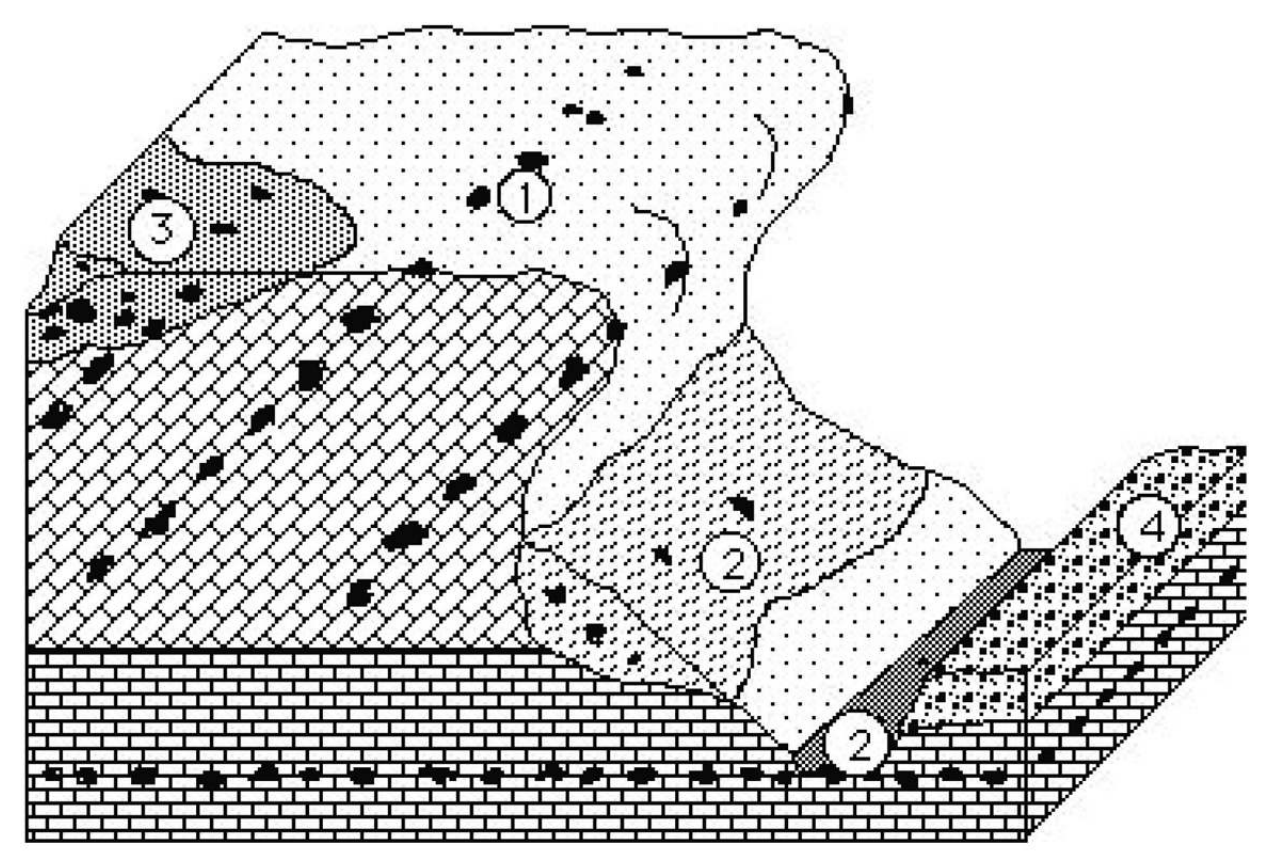

1, autochtone primaire ou in situ (en place dans la roche mère) ; 2 , autochtone secondaire (dans un dépôt de pente) ; 3, sub-allochtone ou résiduel (altérites ou colluvions " sidérolithique ») ; 4, allochtone (ayant parcouru de longues distances dans des formations alluviales).

7, primary source or in situ (in locations with parent rock) ; 2, secondary source (in a slope deposit) ; 3 , residual context (weathered and decayed rock or colluvium "sidérolithique") ; 4, exotic (transported long distances to the alluvial deposit).

\section{Les travaux de terrain (l'inventaire des gîtes)}

Dans la plupart des travaux, l'ensemble des types de gîtes n'est pas pris en compte. Certains auteurs n'ont traité que les silex in situ (Séronie-Vivien 1987), d'autres y ont ajouté les silicifications des altérites et des dépôts de pente dérivés (Demars 1980 ; Morala 1980 ; Geneste 1985 ; Turq 1992). Quant aux alluvions, le plus souvent, elles ne sont qu'évoquées. L'objectif premier qui est de donner l'image la plus fidèle des ressources actuelles n'est donc pas atteint. De plus, l'échelle de prospection et de prélèvement diffère beaucoup d'un individu à l'autre. Certains ont cherché à avoir une vision générale et la maille est lâche. Pour d'autres ayant des préoccupations microrégionales, elle est plus serrée. Même dans le Nord-Est aquitain qui est étudié depuis plus 25 ans, il reste beaucoup à faire. Quelques exemples permettent de s'en rendre compte.

\section{A - Gîtes primaires}

19 Pour les silicifications en place dans la roche mère, dont les positions stratigraphiques sont bien connues ${ }^{4}$ et les plus faciles à répertorier puisque nécessitant un simple suivi des affleurements dont les principaux sont connus. Dès que nous passons à l'échelle 
micro-régionale (celle qui nous intéresse dans le cadre de l'étude d'un gisement archéologique), les lacunes apparaissent :

1. des rides anticlinales comme celle du Pech-de-l'Azé qui mettent à l'affleurement des niveaux à silex et ont favorisé le développement de cavités karstiques utilisées par l'Homme sont passées inaperçues (Turq et al. 1999). Sur la rive droite de la vallée de la Dordogne, la cartographie des niveaux à silex de la base du Coniacien s'arrête là où s'interrompt la cartographie de ces formations sur la carte géologique. En fait, ils affleurent encore plusieurs kilomètres vers l'est.

2. par ailleurs, dans toutes les formations à silex du Sénonien, outre les silicifications classiques, il existe dans tous les étages des nodules de silex calcédonieux (certains de plusieurs kilogrammes) qui sont le plus souvent passés inaperçus. Les objets fabriqués dans ces matériaux ont fréquemment été considérés comme provenant de l'exploitation de silex tertiaires (fig. 4).

Figure 4 - Silex de type calcédonieux. Géode de calcédoine mamelonnée formée dans un nodule de silex santonien, altérite de la région de Tamniès (Dordogne), (Récolte A. Turq, cliché Ph. Jugie,

M.N.P.).

Figure 4 - Calcedonic flint in a geode containing mamillated chalcedony formed within a santonian flint nodule, weathered and decayed rock, Tamniès region (Dordogne), (Collected by A. Turq, photographed by Ph. Jugie, M.N.P.).

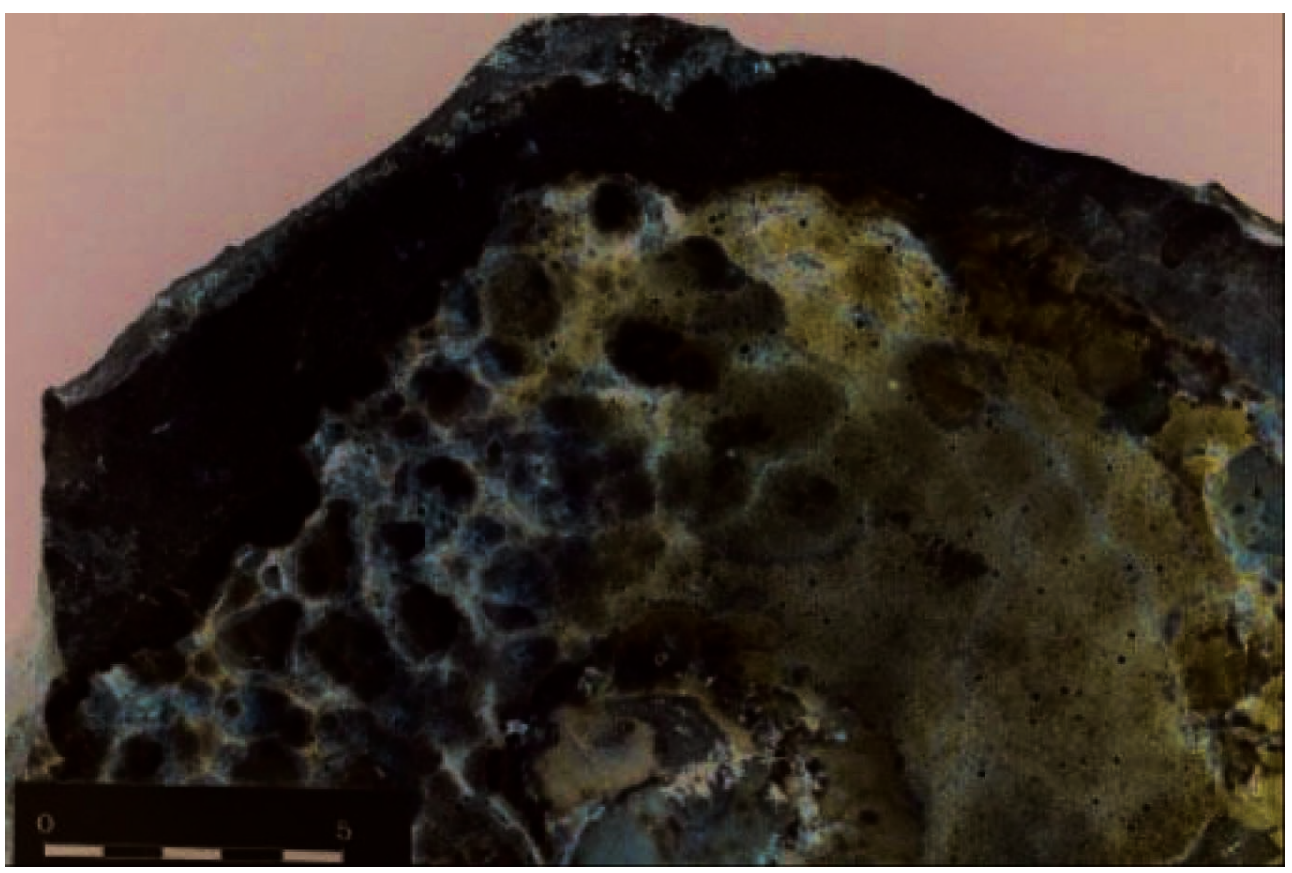

\section{B - Gîtes inclus dans les altérites}

20 En ce qui concerne les gîtes des altérites, l'importance du couvert végétal a considérablement gêné leur cartographie. Aussi, nous ne connaissons qu'une part infime de leur potentiel, alors que ces formations renferment des silicifications particulièrement importantes dont des silcrètes. Les phénomènes physico-chimiques subis dans ces milieux par les blocs ne sont pas homogènes et donnent une diversité de caractères qui sont susceptibles d'aboutir à des matériaux spécifiques (voire caractéristiques) et/ou, au contraire, à des types très comparables avec d'autres connus dans d'autres contextes stratigraphiques, géographiques ou géologiques. L'une de leurs caractéristiques est d'ordre colorimétrique: ils présentent une grande diversité de 
couleurs vives (fig. 5) et souvent des zonations. L'un des types assez fréquent, dit à zone sous-corticale rouge, présent dans les altérites du Santonien du bassin hydrographique de la Lémance (silex de la Séguine, Turq 1977a) mais aussi du nord-est de Périgueux (Demars 1980) peut d'abord être confondu avec du Bergeracois. D'autres à périphérie rouge brique ou lie de vin, ont parfois un cœur de couleur moutarde moucheté de noir qui rappelle alors certains jaspes de la région de Brive (Turq 1979b : p. 224).

Figure 5 - Silex des altérites du Santonien du Haut-Agenais. On note les couleurs vives acquises dans les altérites très riches en fer (récoltes A. Turq, cliché Ph. Jugie, M.N.P.).

Figure 5 - Santonian flint in sediments derived from parent rock at Haut-Agenais : Note : Vibrant colours can be observed on flint found in iron-rich sediments (Collected by A. Turq, photographed by Ph. Jugie, M.N.P.).

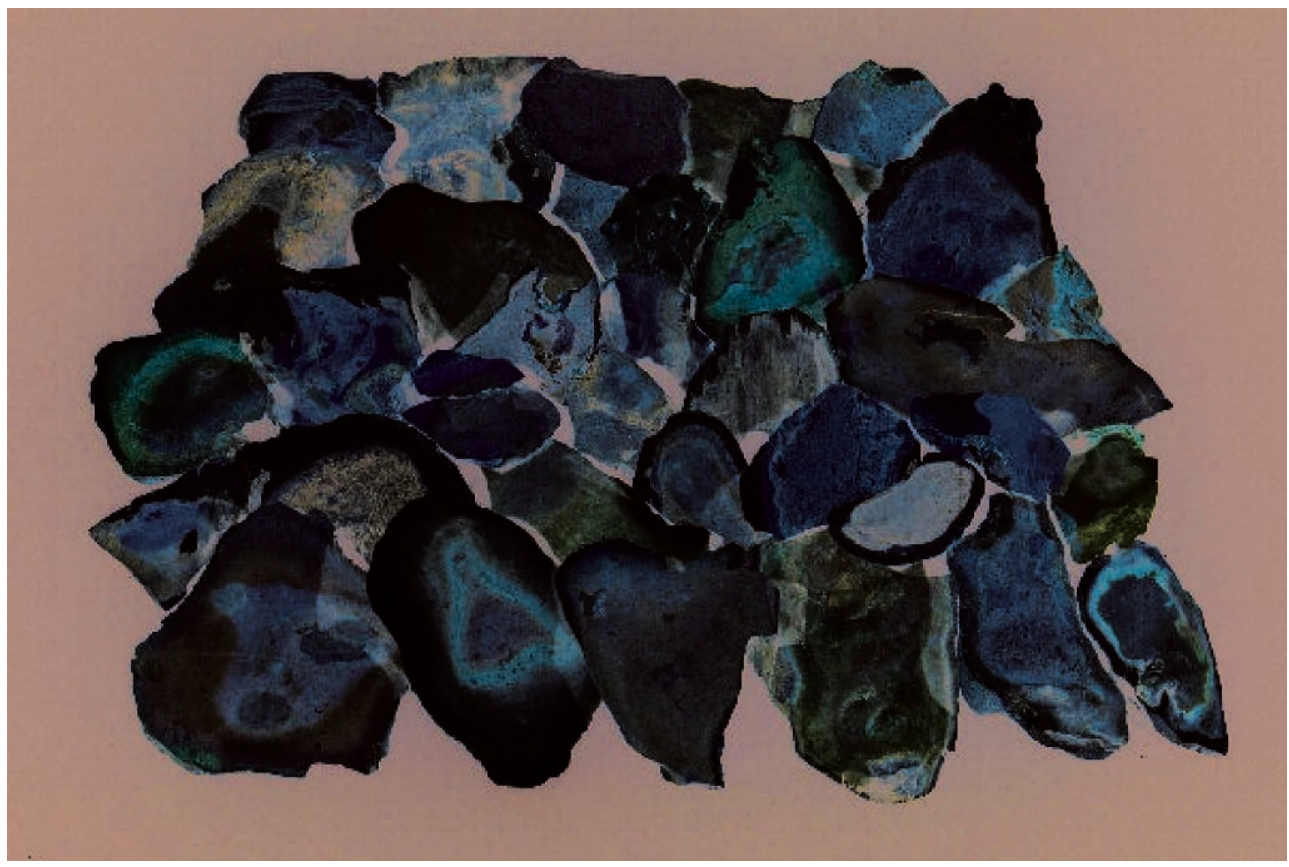

\section{C-Gîtes alluvionnaires}

21 Les gîtes des alluvions (tout comme ceux des cordons littoraux) ${ }^{5}$ n'ont pas fait l'objet de travaux spécifiques et, tout au plus, ont-ils été pris en compte d'un point de vue cartographique (Turq 1992). De fait, ils ont donc, le plus souvent, été oubliés ou sousestimés lors des phases interprétatives. Par exemple, dans la vallée de la Dordogne, la méconnaissance de leur contenu a donné lieu à des interprétations discutables: les jaspes de l'Hettangien et les silex lacustres de type bordure du Massif central (Turq 1992) "type bassin d'Aurillac » proviennent des affleurements primaires (Rigaud 1982 ; Soressi 2002 pour les premiers, Chiotti et al. 2003 ; Lucas 2000 pour les seconds) alors que ces silicifications se trouvent dans les alluvions de la Dordogne. Les silex des PréPyrénées et des anticlinaux du sud du Bassin aquitain caractérisés par la présence de Lepidorbitoides $s p$. issus des formations recoupées et draînées par les affluents de la rive gauche de la Garonne proviendraient-ils toujours du pied de la chaîne ou des anticlinaux de Chalosse (Lenoir et al. 1998 ; Séronie-Vivien 2003 ; Le Brun Ricalens et Séronie-Vivien 2004) alors que des galets ont été trouvés dans la Baïse et existent donc, au moins potentiellement, en aval dans les alluvions de la Garonne? 
La méconnaissance du potentiel des alluvions de la Vézère a permis d'envisager l'épuisement de ce type de gîte (Demars 1990, 1994) alors que celui-ci n'est pas concevable pour au moins deux raisons: leur richesse en silex (100 000 rognons à l'hectare) et leur renouvellement lors des crues (reprises de galets sous-jacents et de ceux situés en amont).

\section{D - Extensions géologiques maximales}

Enfin, notons que les géologues (Platel 1983 ; Kafa 1988 ; Astruc 1988, 1990 ; SimonCoinçon et Astruc 1991) ont signalé qu'à l'origine, les formations sédimentaires du Bassin aquitain avaient une extension bien plus importante vers l'est. Ceci est confirmé par la présence de silex crétacés dans les alluvions de hautes terrasses du Lot bien en amont des affleurements actuels (Séronie-Vivien 1987; Turq 1992) ${ }^{6}$. Pour certains auteurs (Séronie-Vivien. 1995), il peut rester ponctuellement des formations relictuelles avec des silex comme par exemple le silex du Fumélois. Ce point essentiel qui a évidemment des incidences sur l'interprétation technologique voire socioéconomique des ensembles archéologiques, doit également être pris en compte. Par précaution et avant que des travaux longs et fastidieux ne permettent de préciser l'importance de ce phénomène. Il serait bon de garder en mémoire ces possibilités d'extension géographiques lors de la cartographie des ressources potentielles en silex (Turq 1992).

\section{La cartographie}

Elle doit considérer l'ensemble des ressources actuelles, c'est à dire tous les types de gîtes. Si tel n'est pas le cas, il peut y avoir des distorsions importantes (fig. 6). Dès 1992, il nous est apparu important d'ajouter les alluvions qui se sont révélées être parmi les gîtes les plus fréquentés par les hommes paléolithiques, mais aussi de signaler, par précaution, la possibilité de présence de formations relictuelles. 
Figure 6 - Cartographie des silicifications du Santonien. Figure 6 - Map showing the distribution of Santonian flint.
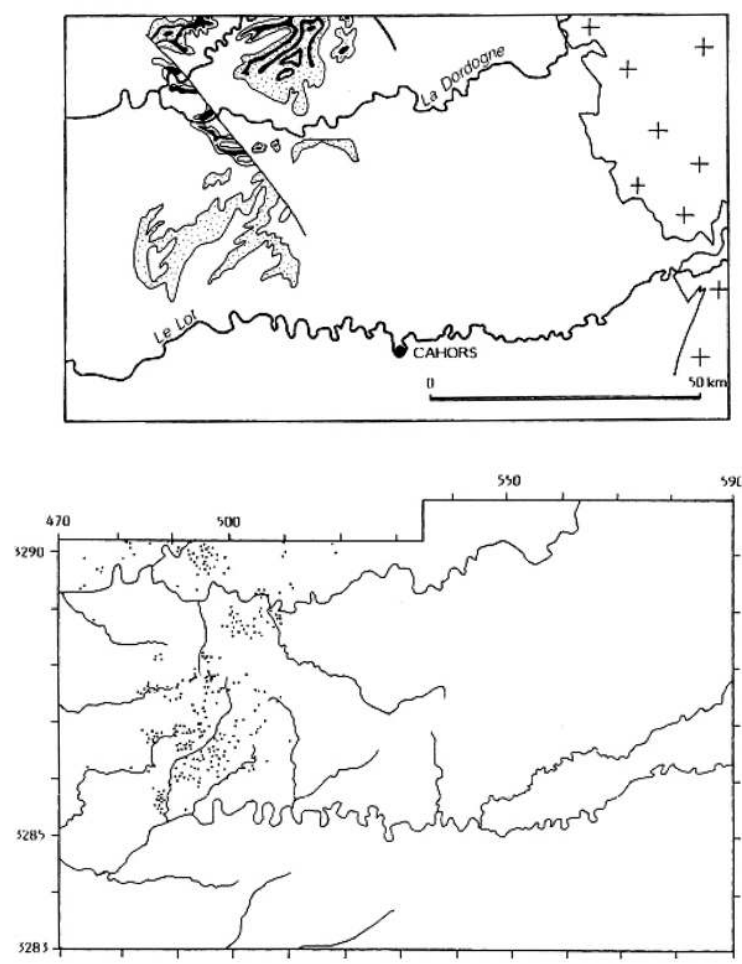

En trait plein les affleurements in situ (d'après M.R. Séronie-Vivien 1987) ; les points correspondent aux gîtes des altérites (d'après Geneste 1985 et Turq 1992) ; la zone en grisé à la zone d'extension maximum supposée de cette formation (Turq 1992).

Unbroken line represents in situ flint (after M.R. Séronie-Vivien 1987); dots show the location of sources in altered formations (after J.-M. Geneste 1985 et A. Turq 1992); the grey zone represents the estimated maximum extent of this formation (A. Turq 1992).

\section{Les fiches d'inventaire}

Très tôt, des fiches de recensement des gîtes potentiels de matières premières lithiques ont vu le jour (Malissen 1977). Toute une série d'autres paramètres, qui différent selon les auteurs, ont été retenus : données cartographiques, bibliographiques, informations concernant les gîtes, les silicifications (quantité, qualité, dimensions, morphologie...), la présence ou l'absence de traces d'exploitation. Ces documents souvent fastidieux à compléter et lourds à gérer n'ont pas été exploités ou alors très partiellement.

\section{2 - Des propositions pour une meilleure estimation des ressources}

Des méthodes simples permettent d'obtenir des données mieux adaptées aux besoins des études litho-technologiques. Outre une cartographie plus fine des gites, il convient de mieux estimer qualitativement et quantitativement les ressources.

Pour atteindre cet objectif, trois critères doivent être retenus: les dimensions, la morphologie et l'aptitude à la taille. Pour ce denier point, nous avons opté pour une méthode simple permettant de donner les premiers éléments d'une estimation raisonnée de l'aptitude à la taille. Connaissant les difficultés à mettre en évidence des 
caractères objectifs de caractérisation et sachant que la solution du problème passe par une nécessaire étude collective faite par des expérimentateurs, nous nous sommes engagé dans un système simple et transitoire. Les tests systématiques ont été faits au percuteur dur. Trois groupes ont été individualisés :

1. les mauvais silex qui sont très hétérogènes (vacuolaires ou saccharoïdes), fracturés, clivés. Ils sont pratiquement impropres à la taille, toutefois on ne peut exclure la possibilité de la production de quelques éclats ;

2. les silex de bonne qualité sont plus homogènes. Ils permettent des productions d'éclats en série et le façonnage de bifaces. Leur qualité ne permet pas, à coup sûr, la production systématique de supports Levallois, de lames ou le façonnage de feuilles de laurier ;

3. la catégorie des très bons matériaux permet toutes les méthodes de production de supports et de façonnage par percussion et probablement la retouche par pression?

Deux exemples montrent tout l'intérêt de cette démarche.

\section{Estimation des gîtes des altérites}

Dans les cartographies actuelles, tous les gîtes présents dans les altérites du Santonien de la vallée de la Vézère sont matérialisés de façon identique. Si nous savions globalement que les silicifications sont de meilleure qualité dans le secteur de Tamniès que vers Rouffignac (Demars 1980 ; Geneste 1985), il était nécessaire de préciser les différences. Deux gîtes des plateaux dominant la Vézère ont été choisis (fig. 7) pour montrer l'ampleur et l'implication des différences observables dans les altérites d'une même formation : l'un est situé sur la commune de Fleurac (A) et l'autre sur celle de Tamniés (B). Tous deux livrent des rognons principalement oblongs ainsi que des plaquettes. Sur chacun d'eux une centaine de nodules ont été recueillis, mesurés et testés. 
Figure 7 - Situation géographique des gîtes étudiés. Figure 7 - Geographic location of the studied sources.

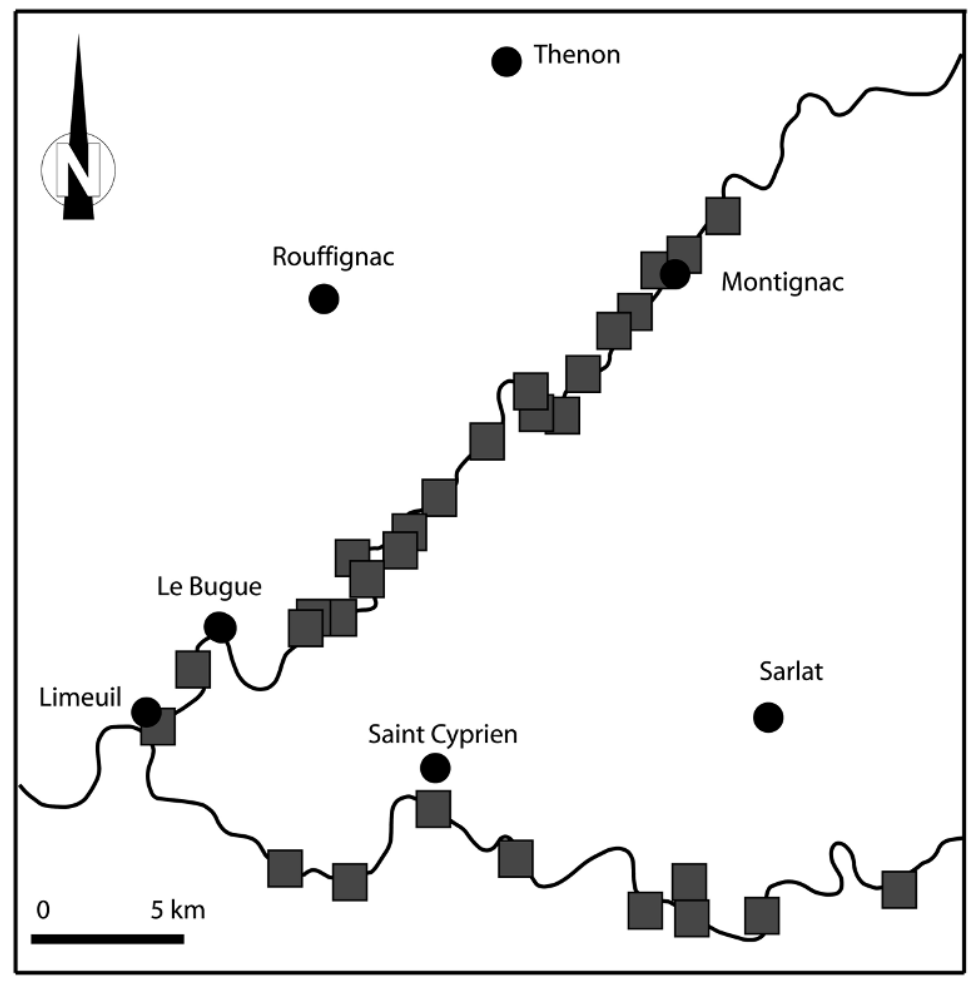

Les carrés correspondent aux prélèvements dans le lit de la Dordogne et de la Vézère ; les cercles montrent l'emplacement des villages et des villes.

Squares represent studied samples from gravels above the high water mark in the Dordogne and Vézère Rivers; circles show the location of villages and cities.

La figure 8 montre que le gîte A livre principalement des silex impropres à la taille et le gîte B un matériau de bonne qualité. D'un point de vue morphométrique (fig. 9), le gîte A contient surtout des silex de dimension petite à moyenne (entre 10 et 25 centimètres) alors que le gîte de Tamniès possède des blocs de dimension grande pour le Périgord (entre 20 et 30 centimètres).

31 L'examen des vestiges archéologiques attribuables au Paléolithique inférieur ou moyen, récoltés sur chacun d'eux, confirme les possibilités estimées actuellement. A Fleurac, seules quelques pièces et de rares éclats ont été retrouvés. A Tamniès, les vestiges sont très nombreux et des débitages tout à fait exceptionnels pour le Périgord sont représentés: un large éventail de méthodes Levallois avec notamment des grands nucléus à éclats préférentiels, à pointes et plusieurs débitages laminaires. Cet exemple montre que la cartographie généralement pratiquée n'est pas ou peu représentative de la réalité des ressources actuelles. Les expérimentateurs le savent puisque, par souci d'efficacité, ils ne s'approvisionnent que sur un nombre limité de gîtes, ceux qui livrent les matériaux les mieux adaptés à leur besoin. Ne peut-on pas penser qu'il en était de même pour l'Homme préhistorique? 
Figure 8 - Données qualitatives concernant les gîtes des altérites de Tamniés et de Fleurac. Figure 8 - Differences in the quality of altered flints from deposits at Tamniés and Fleurac.

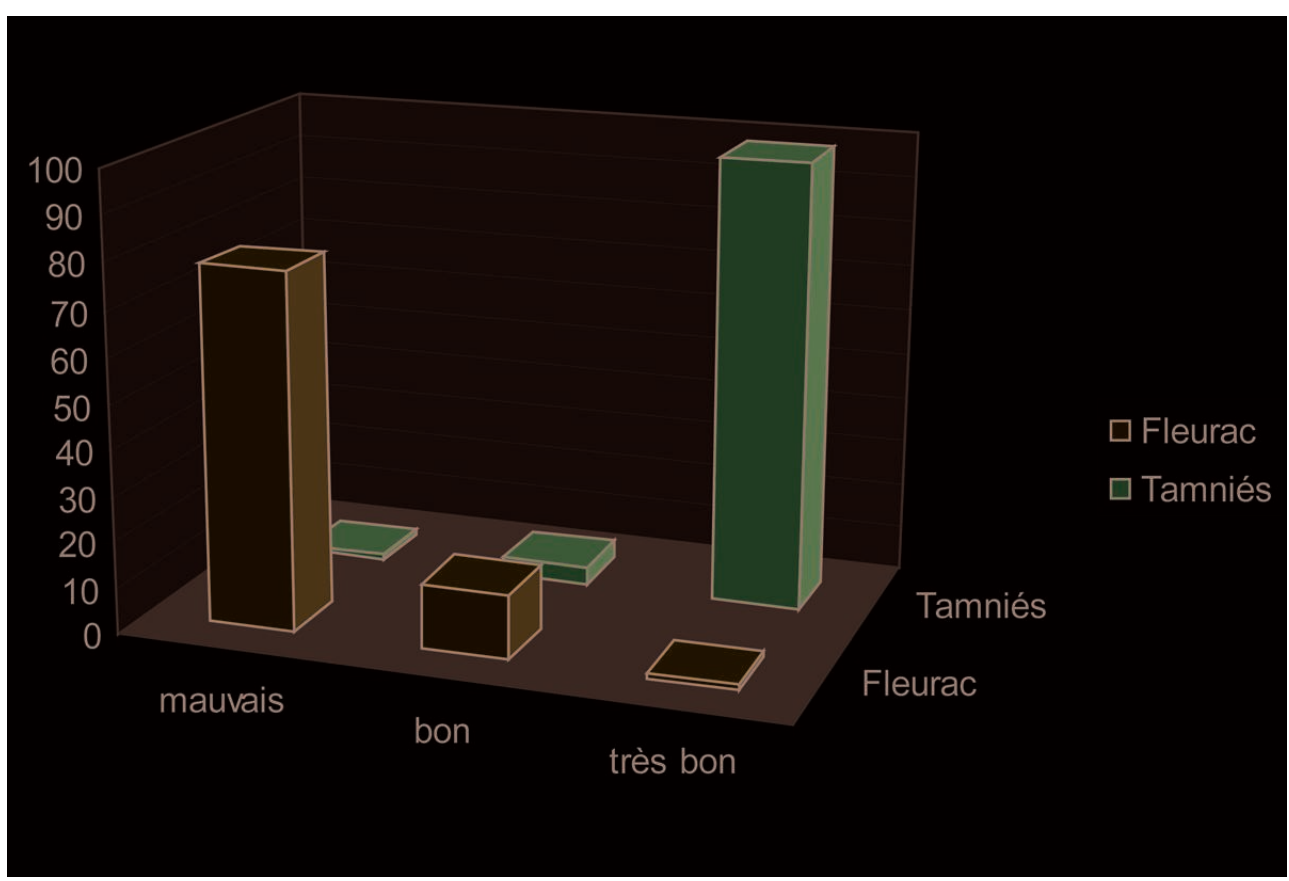

103 blocs ont été testés sur le premier et 93 sur le second. 103 blocks tested at the first and 93 blocks at the second

Figure 9 - Données dimensionnelles concernant les gîtes des altérites de Tamniés et de Fleurac. Figure 9 - Differences in the dimensions of altered flints from deposits at Tamniés and Fleurac.

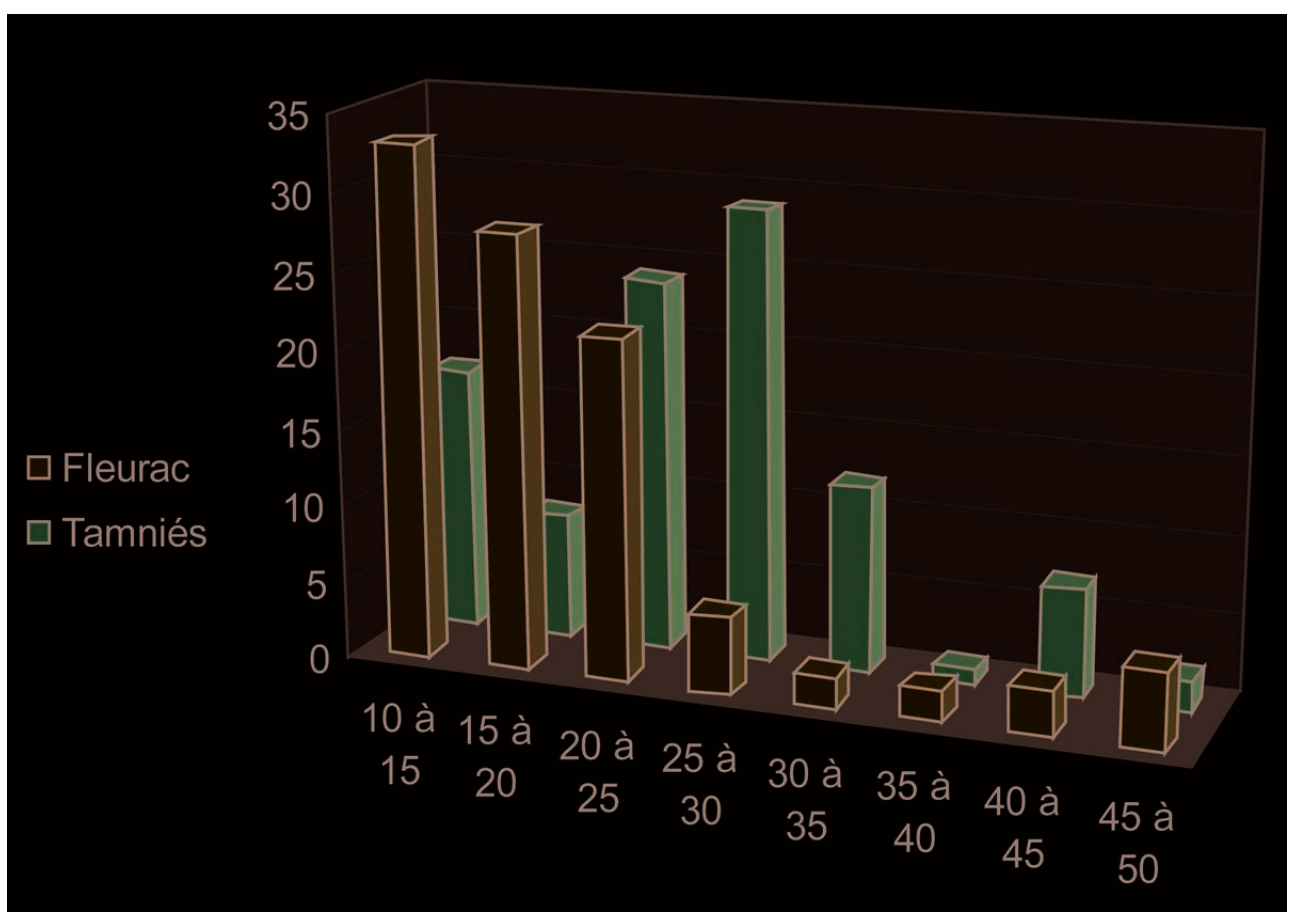

103 blocs ont été mesurés sur le premier et 93 sur le second. En abscisse, les mesures déterminant les classes sont exprimées en centimètres.

103 blocks tested at the first and 93 blocks at the second. Horizontal axis shows the size classes in centimètres. 


\section{Estimation du potentiel des alluvions récentes de la Dordogne et de la Vézère}

leurs alluvions. Les alluvions de fond de vallée de la Vézère actuelle comportent 4 fois plus de silex que ceux de la Dordogne (tabl.2). Les cortèges de silicifications sont globalement comparables (tabl.3) mis à part la présence dans la seule vallée de la Dordogne de silicifications tertiaires provenant du Massif central ou de sa bordure. Pour ce qui est de l'aptitude à la taille, les galets de silex sont majoritairement de bonne à très bonne qualité pour la Vézère et de mauvaise qualité pour la Dordogne (tabl. 4).

Tableau 2 - Composition lithologique globale des alluvions actuelles. Table 2 - Total lithic composition of present gravels.

\begin{tabular}{|l|l|l|l|l|l|}
\hline & Quartz & Calcaire & Silex & Roches éruptives & Nombre de galets examinés \\
\hline Dordogne & $7-9 \%$ & $1-3 \%$ & $0-5 \%$ & $75-88,7 \%$ & 2524 \\
\hline Vézère & $43-75 \%$ & $0-6 \%$ & $4-20 \%$ & $11-39 \%$ & 1852 \\
\hline
\end{tabular}

Les deux chiffres correspondent aux pourcentages minimum ou maximum rencontrés dans l'ensemble des prélèvements effectués sur chacune des rivières.

Tableau 3 - Origine géologique des silex. Table 3 - Geological origine of flints.

\begin{tabular}{|l|l|l|l|l|l|l|}
\hline & Sénonien & Jurassique & Tertiaire & Tertiaire du Massif central & Indét. (chaille) & Jaspe \\
\hline Dordogne & $65 \%$ & $0,6 \%$ & $17 \%$ & $1,7 \%$ & $19 \%$ & $0,6 \%$ \\
\hline Vézère & $99,1 \%$ & $0,6 \%$ & $1 \%$ & $0 \%$ & $0,1 \%$ & $0,1 \%$ \\
\hline
\end{tabular}

Tableau 4 - Aptitude à la taille des blocs. Table 4 - Knapping ability of blocks.

\begin{tabular}{|l|l|l|l|}
\hline & Mauvaise & Bonne & Très bonne \\
\hline Dordogne & $64 \%$ & $25 \%$ & $11 \%$ \\
\hline
\end{tabular}




\begin{tabular}{|l|l|l|l|}
\hline Vézère & $34 \%$ & $36 \%$ & $30 \%$ \\
\hline
\end{tabular}

34 Pour la vallée de la Vézère, particulièrement riche en galets de silex, nous avons analysé plus en détail 1100 nodules de silex du Sénonien. Les gris ou noirs et les blonds sont en proportions presque identiques, (respectivement $51,5 \%$ contre $48,5 \%)^{8}$.

Pour ce qui est de la morphologie des blocs ont été retenues en dehors des artefacts toujours présents, les plaquettes, les formes contournées, oblongues, globuleuses ou «patatoïdes » et les branchues. Comme le montre la figure 10 il n'y a pas de grandes différences entre les deux grands types.

Figure 10 - Alluvions du lit mineur de la Vézère.

Figure 10 - Gravels above the low flow channel of the Vézère River.

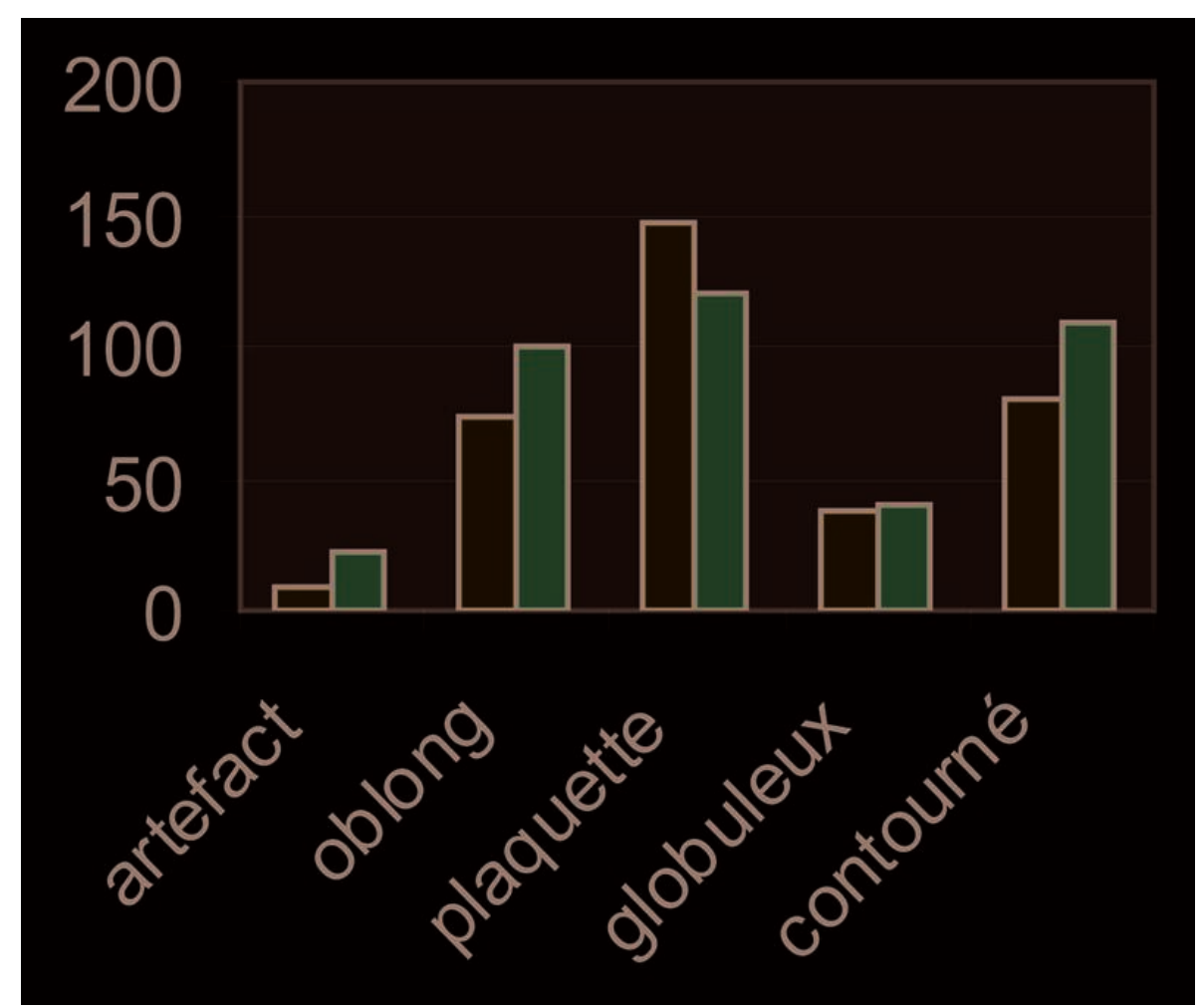

Morphologie des silex gris ou noirs du Sénonien et des silex Blonds du Sénonien (731 blocs). Horizontal axis shows variations in the morphology of beige and grey and/or black Senonian flint (737 blocks studied).

Les dimensions sont principalement comprises entre 9 et $16 \mathrm{~cm}$ (fig. 11), ce qui n'exclut pas que quelques blocs puissent dépasser les 40 centimètres ${ }^{9}$. 
Figure 11 - Alluvions du lit mineur de la Vézère.

Figure 11 - Gravels above the low flow channel of the Vézère River.

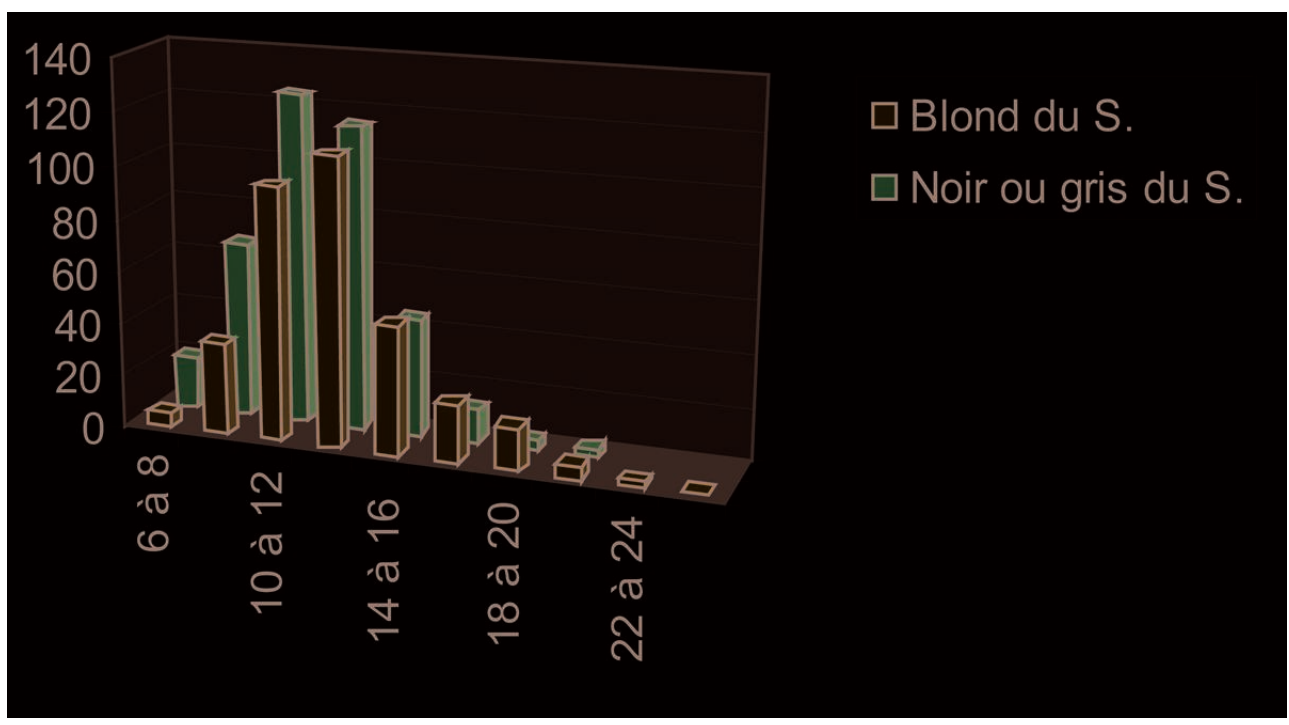

Dimensions respectives des silex gris ou noirs du Sénonien et des silex Blonds du Sénonien (731 blocs).

Horizontal axis shows the size classes in centimetres for beige and grey and/or black Senonian flint (737 blocks studied).

En terme d'aptitude à la taille, les galets de silex gris et noirs sont bien meilleurs que les blonds (fig. 12), quelles que soient leur morphologie et leur dimension. Ces données sont le reflet fidèle des observations faites dans les gites du bassin hydrographique. Les silex gris ou noirs provenant surtout des gîtes in situ sont de meilleure qualité que les silex blonds issus principalement des altérites du Santonien qui, comme nous l'avons vu précédemment, sont très hétérogènes.

Figure 12 - Qualité respective des silex gris ou noirs du Sénonien et des silex blonds du Sénonien (731 blocs), dans les alluvions du lit mineur de la Vézère.

Figure 12 - Differences in the quality of beige and grey and/or black Senonian flint from gravels above the low flow channel of the Vézère River (731 blocks tested).

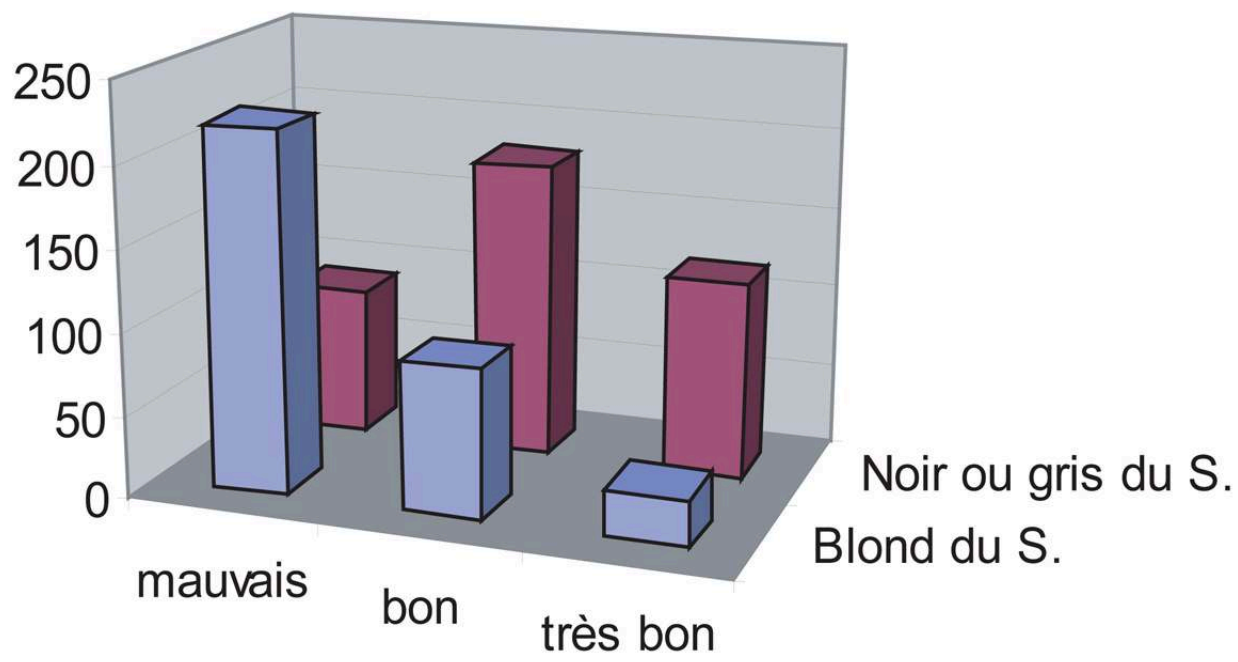



évidence des distorsions importantes entre les données recueillies, les attentes issues de l'observation du bassin versant et les résultats des études archéologiques :

1. dans la vallée de la Vézère, les prélèvements faits dans les alluvions ne reflètent pas la totalité du potentiel du bassin versant. On note une sous-représentation des silex des altérites de la rive droite (localisées autour de Thenon, Rouffignac, Mauzens, Miremont), en particulier des silex zonés ou à zone sous-corticale rouge et le silex de type Sainte-Foy, caractéristique de la base du Campanien (Turq 1992).

2. pour la Dordogne, les gîtes actuels sont d'un faible d'intérêt archéologique, ce qui est incompatible avec les données archéologiques issues du gisement de Combe-Grenal (Bordes F. 1972.). Aujourd'hui, dans ce secteur, les plages fournissent un à deux rognons de silex sénoniens à l'hectare, alors que dans certaines couches de ce gisement les mêmes silex, à cortex fluviatile, représentent des pourcentages significatifs (plus de $50 \%$ ). Il existe donc une différence importante entre les ressources actuelles et celles du Würm ancien. Ces distorsions observées confirment une nouvelle fois que les données recueillies sur les ressources lithiques actuelles ne sont pas directement transposables et exploitables dans des études traitant de sites paléolithiques.

\section{3 - Conclusion}

Les lithothèques qui se veulent et doivent être le reflet le plus exact possible du potentiel actuel ont encore des progrès à faire pour atteindre leur objectif premier, caractériser les matières premières disponibles et estimer le potentiel lithique de chaque territoire. Lorsque celui-ci sera atteint, seule la première étape de la recherche sera franchie. En effet, "... tout inventaire de gîtes, aussi exhaustif soit-il, ne sera qu'une image déformée des possibilités d'approvisionnement paléolithiques" (Chadelle 1983, p. 28). La seconde phase indispensable sera d'essayer d'estimer les ressources au moment de l'occupation préhistorique étudiée. C'est seulement ensuite que l'analyse de l'origine des matières premières utilisées dans un ensemble archéologique deviendra plus fiable.

\section{3 - De l'échantillon de la lithothèque au matériel archéologique}

41 Chaque série analysée a ses propres particularités en terme d'état de conservation et d'âge estimé. Avant donc de passer à l'interprétation, il convient de prendre en compte ces deux éléments. Le matériel archéologique plus ou moins altéré extérieurement doit être comparé avec l'échantillon frais.

\section{1 - Du silex frais au matériel archéologique}

Comme nous l'avons vu précédemment, des altérations anthropiques ou naturelles affectent généralement le matériel lithique. Les altérations anthropiques, la chauffe accidentelle ou volontaire, peut être totalement absente ou affecter une partie du matériel dès le Moustérien avec $4 \%$ dans la couche B du Pech de Bourre (Turq inédit), 
$11 \%$ dans la couche de base du Pech de l'Azé IV (Dibble, MacPherron 2001), 19,3\% dans la couche III du Roc de Marsal (Thiébaut 2003) et atteindre $50 \%$ au Mésolithique (Séronie Vivien 2001). Elle amène une modification au moins de la couleur (zone bleutée ou blanchâtre) et parfois de la structure (apparition d'un réseau de fissures, de craquelures ou de cupules thermiques).

Les altérations naturelles post-dépositionnelles sont aussi très fréquentes. Il peut s'agir de lustrage ou de concassage mais, le plus souvent, de patine pouvant aller du simple voile gênant l'approche à une attaque profonde qui peut empêcher toute étude.

Pour tenter de pallier ces difficultés, il convient de compléter la lithothèque de référence des matériaux frais par :

1. des échantillons traités thermiquement ${ }^{10}$;

2. des objets patinés. L'idéal serait de trouver des objets patinés sur une seule face comme on l'observe souvent dans certains sites de plein air. A défaut, on peut prendre des objets dont toute la surface est altérée et les casser pour aboutir à une identification sur la partie non altérée. La multiplication de ces échantillons permet d'analyser l'altération d'un type de silex et éventuellement mettre en évidences des critères d'identification: uniformité de l'altération, modification de seulement quelques éléments... Il y a déjà plus de vingt ans que, comme d'autres chercheurs, nous avons recours à cette démarche.

\section{2 - De l'évaluation des ressources actuelles à l'estimation des ressources passées}

Le résultat de notre travail sur les ressources actuelles aboutit in fine à une cartographie qui, une fois publiée, est un document figé. Chaque analyse lithologique nous amène à estimer les ressources telles qu'elles pouvaient se présenter au moment de l'occupation étudiée. Ceci nécessite la prise en compte de l'évolution géologique régionale et une bonne connaissance des conditions climatiques qui prévalaient au moment de l'occupation considérée.

Plus nous remontons dans le temps, plus le transfert des données concernant les ressources actuelles est difficile. Voici quelques exemples pour illustrer le propos.

Sur le site du Roc-Allan (Coulonges 1935,1963 ; Le Tensorer 1979 ; Turq 1987-1988), un changement dans l'approvisionnement en matières premières lithiques entre les occupations magdaléniennes, aziliennes d'une part et celles du Sauveterrien et du Néolithique ancien d'autre part, a été constaté (Turq et al. 1996). Les premiers utilisent surtout les silex des dépôts de versant carbonatés et des alluvions, les second ceux des altérites des plateaux environnants. Cette modification de comportement correspondant aussi à un changement culturel, il est important de savoir si l'observation faite est à mettre en relation avec un changement lié à l'Homme ou à des problèmes d'accessibilité aux gîtes.

Les données recueillies permettent de retenir la seconde solution. Les dépôts de pente carbonatés anciennement fréquentés sont recouverts et scellés par la végétation (entre autre le noisetier) et ceux des terrasses alluviales recouverts par un remplissage détritico-organique lié à une modification de l'écoulement des cours d'eau (Turq et al. 2000). Quant aux gîtes des altérites, malgré le couvert végétal, ils restent partiellement accessibles sans doute à la faveur de coulées boueuses, ravines, chablis et autres. 
Le principe de fonctionnement mis en évidence ici a été retrouvé dans d'autre sites synchrones comme La Borie del Rey (Coulonges 1963), Le Martinet (Coulonges 1935) et le Pont d'Ambon (Célérier 1998), tous implantés dans des vallées secondaires des terrains sénoniens $\mathrm{du}$ nord-est $\mathrm{du}$ Bassin aquitain. En outre, l'apparition et le développement $\mathrm{du}$ Castor dans la faune de ces sites viennent confirmer le remblaiement des fonds de vallées et la fin de l'accessibilité des gîtes situées dans les alluvions des vallées secondaires.

Si dans un laps de temps relativement court, à l'échelle de la Préhistoire (10 000 ans) l'accessibilité de certains gites s'est autant modifié on comprend qu'il est encore plus délicat d'estimer les ressources pour des périodes beaucoup plus anciennes. Prenons comme exemple la découverte, sur le tracé de l'autoroute A20 (Jarry et al. 2001), du site des Bosses (commune de Lamagdeleine) daté d'environ 300000 ans. Ce gisement implanté sur la rive droite du Lot, à la surface de la moyenne terrasse, a livré une industrie réalisée majoritairement en métaquartzite comprenant néanmoins près de 400 objets en silex. Si la patine rend non identifiable l'origine de $14 \%$ d'entre eux, les autres comprennent (Chalard, Turq 2001) des silex tertiaires $84 \%$, des silex jurassiques (11\%), des silex crétacés $(3 \%)$ et des silex jaspoïdes $(1 \%)$. Si pour la majorité d'entre eux, l'origine fluviatile ne fait aucun doute, la question se pose pour quelques pièces en silex tertiaire et crétacé ne présentant pas de néo cortex. D'où viennent ces matières premières? Des lambeaux des hauts niveaux fluviatiles du Lot, du corps de la terrasse sous-jacente, de lambeaux de formations disparues ou des affleurements actuels? Si, d'un point de vue numérique la réponse ne modifie que faiblement la diagnose (approvisionnement local), la présence ou l'absence de silex importés sur plusieurs dizaines de kilomètres est plus importante car elle identifie ou non un territoire d'acquisition développé vers le Périgord et le Haut-Agenais. La solution passe forcément par la prise en compte de formations sédimentaires aujourd'hui disparues mais repérables par leurs éléments retrouvés dans les formations alluviales ( $c f$. supra) et par l'étude détaillée du contenu lithologique de chacun des niveaux des terrasses. Des silex crétacés existent dans les niveaux de haute terrasse près du site.

Figure 13 - Cartographie des affleurements actuels des formations crétacées (hachures), localisation des hautes terrasses ayant livré des silex crétacés (toutes celles en aval du confluent avec le Célé).

Figure 13 - Map showing the actual distribution of Cretaceous formation (delimited by the broken line in the upper left), black areas show the location of high terraces where cretaceous flint can be found (always down stream from the junction of the Célé and Lot Rivers).

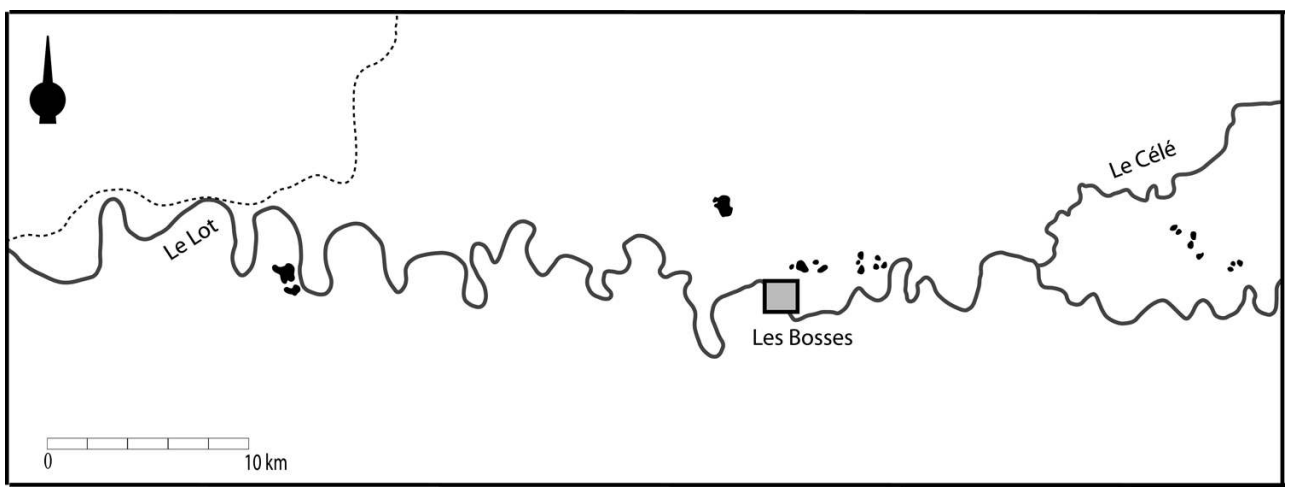

Le carré matérialise le site des Bosses.

The square is the site of Bosses. 
Ces observations confirment une nouvelle fois que la cartographie actuelle des ressources n'est pas directement utilisable. Les sources de matières premières lithiques accessibles ont changé en fonction de l'évolution géomorphologique régionale: disparition par érosion de certains affleurements d'altérites, apparition de nouveaux horizons à silex mis à nu par l'incision des vallées... Dans l'attente de longs travaux de géomorphologie (étude détaillée de l'évolution des paysages), les premières approches peuvent s'appuyer sur la prise en compte de l'évolution du réseau hydrographique : le système de terrasses étagées peut servir de base morpho-chronologique globale. L'approche repose sur le croisement des données théoriques (ressources du bassin versant) et les grandes lignes de l'évolution du modelé des versants déduites de l'incision des cours d'eau et du contenu des différentes nappes de terrasses. Les ressources du bassin versant correspondent aux gites autochtones (primaires) et suballochtones (secondaires) actuels recensés. Pour ce qui est de l'évolution, elle peut être estimée à partir des facteurs susceptibles de faire varier ces ressources. L'accès aux gites autochtones ou primaires dépendent principalement de l'incision de l'affleurement par le réseau hydrographique. En effet, lorsqu'il s'agit d'affleurements continus sur plusieurs kilomètres, il est difficilement concevable que les dépôts de versant puissent en interdire totalement l'accès. Du fait d'un encaissant généralement massif, l'érosion n'a que peu d'emprise sur eux. Les gîtes sub-allochtones ou secondaire proche sont plus fragiles : le remplacement de la roche-mère par du sable ou de l'argile les rend vulnérables. D'une part, les blocs sont davantage soumis à des agressions physiques (chocs thermiques, mécaniques) ou chimiques (remobilisation de la silice, imprégnations...) et d'autre part, l'affleurement évolue rapidement au gré des changements de climat et/ou de la présence ou l'absence d'un couvert végétal ( $c f$. supra), facteurs capables de pouvoir rapidement le réduire ou le détruire. Les gîtes allochtones ou secondaires éloignés sont, quant à eux, tributaires des facteurs influents sur les précédents mais aussi des capacités de transport des affluents temporaires ou permanents, et durant le dépôt des alluvions, de la compétence de la rivière. L'essai de synthèse des données (tabl.5) montre combien, en dehors des gîtes autochtones, les propositions sont fragiles. Leur fiabilité diminue au fur et à mesure que l'on remonte dans le temps :

1. pour l'Holocène, l'évolution des fonds de vallées qui implique le recouvrement des alluvions grossières à galets de silex est le phénomène marquant (Texier 1982 ; Turq et al. 2000);

2. pour le Pléistocène supérieur, les grands changements climatiques sont abordables tant en milieu côtier (variations du niveau de la mer, Monnier 1980, 1988) que continental (Morala et Turq 1990). Pour ce dernier, nous avons pu constater (op. cit.) que certains silex n'affleurant qu'en place dans la roche mère (silex dit de Gavaudun et du Fumélois) ne sont intensément utilisés que lors des phases froides. On peut envisager que le recul des falaises et l'évolution rapide des versants, sous couvert végétal réduit, soient favorables à la régénérescence de ces types de gîtes.

3. pour le Pléistocène ancien et moyen, les modifications du paysage sont telles que la prise en compte des gites aujourd'hui disparus est indispensable (cf. supra). 
Tableau 5 - Stabilité des gîtes et fiabilité de l'estimation des types de gîtes en fonction de l'évolution géomorphologique.

Table 5 - Stability of the different deposits and reliability of their valuation according to the geomorphological evolution.

\begin{tabular}{|c|c|c|c|c|}
\hline $\begin{array}{l}\text { Type de } \\
\text { gîte }^{11}\end{array}$ & Stabilité & Variations liées à & $\begin{array}{l}\text { Estimation dépendante } \\
\text { de }\end{array}$ & $\begin{array}{l}\text { Fiabilité de } \\
\text { l'estimation }\end{array}$ \\
\hline autochtone & maximum & $\begin{array}{l}\text { - l'incision du réseau } \\
\text { hydrographique }\end{array}$ & $\begin{array}{l}\text { - gite actuel } \\
-\mathrm{du} \quad \text { niveau des } \\
\text { terrasses } \\
\text { alluviales }\end{array}$ & bonne \\
\hline $\begin{array}{l}\text { sub- } \\
\text { allochtone }\end{array}$ & moyenne & $\begin{array}{l}\text { - l'érosion } \\
\text { - le climat } \\
\text { - le couvert vétégal }\end{array}$ & $\begin{array}{l}\text { - du gîte actuel } \\
\text { - du contenu des } \\
\text { terrasses alluviales }\end{array}$ & aléatoire \\
\hline allochtone & faible & $\begin{array}{l}\text { - l'incision du réseau } \\
\text { hydrographique } \\
\text { - l'érosion } \\
\text { - le climat } \\
\text { - le couvert vétégal } \\
\text { - les apports des affluents }\end{array}$ & $\begin{array}{lc}\text { - du contenu des } \\
\text { terrasses alluviales }\end{array}$ & médiocre \\
\hline
\end{tabular}

Dans ce domaine beaucoup reste à faire. Si nous voulons essayer de faire la part entre les choix humains et les contraintes naturelles, dans l'approvisionnement en matières premières lithiques, le développement de cet axe de recherche est primordial. L'embryon de réflexion menée ici doit, dans les années à venir, servir de base à un travail d'équipe réunissant géologues, géomorphologues et préhistoriens.

Un autre point important est l'impact climatique sur l'accessibilité aux gîtes. Les années quatre-vingt ont vu les premières études mettant en relation les changements dans les modes d'approvisionnement, les grandes phases climatiques et donc l'accessibilité aux gîtes. Durant les phases froides, il a été observé l'utilisation du silex dans le Paléolithique inférieur et moyen de Bretagne (Monnier 1980 et 1988) et en Haut-Agenais, le rôle accru joué par les silex in situ ou issus des dépôts de pente carbonatés, silex dit de Gavaudun et du Fumélois (Turq 1988a: p. 105; 1989a: p. 186-187; Morala et Turq 1990).

Cet axe de recherche est encore aujourd'hui sous-exploré et mériterait un effort particulier.

\section{4 - Conclusions et perspectives}

La lecture des remarques présentées ci-dessus peut paraitre décourageante. Elle ne doit pas être perçue comme telle, mais plutôt comme une prise de conscience des difficultés et des limites actuelles. L'approche lithologique, en donnant une dimension spatiale, sociale et économique à l'étude du matériel lithique, a ouvert de nouvelles perspectives pour la compréhension du comportement humain préhistorique. Les méthodes utilisées, les outils mis en place demandent, en permanence à être critiqués et 
améliorés. La prise de conscience des problèmes et des limites de notre outil de travail doit être mise à profit pour progresser.

Comme nous l'avons vu, il faut encore et encore améliorer le référentiel des ressources actuelles ainsi que les méthodes de caractérisation pour avoir enfin à disposition l'inventaire « exhaustif » recherché. A la lithothèque des échantillons frais, il convient d'adjoindre des pièces altérées ce qui facilitera les comparaisons et réduira les risques d'erreurs lors du diagnostic.

Une nouvelle étape capitale reste à franchir : mettre en place les outils permettant de passer de l'inventaire des ressources actuelles à l'estimation des ressources dont disposaient les préhistoriques lors de l'occupation du site étudié. Ici, nous n'avons pu aborder que quelques pistes. Le chemin sera long et difficile. Il devra passer par un travail interdisciplinaire avec une meilleure prise en compte des données paléoclimatiques et géomorphologiques. Devant l'ampleur de la tâche et pour ne pas perdre de temps, nous souhaitons proposer des modèles théoriques construits à partir des résultats obtenus lors de l'étude de cas et de les tester pour d'autres sites en essayant de faire varier un ou plusieurs paramètres.

57 Notre principal axe de recherche restera l'accessibilité aux gîtes et plus particulièrement, l'étude des relations entre variations du contexte paléo-climatique et types de gîte exploités. Les résultats des recherches systématiques de deux matériaux (silex dit du Fumélois et silex dit de Gavaudun, non connus dans des gîtes d'altérites) dans l'ensemble des sites du Haut Agenais et du Périgord (donc dans des milieux physiques différents) et la prise en compte des données climatiques a permis d'avancer une hypothèse : l'exploitation préférentielle des gîtes autres que les altérites durant les périodes froides. Aujourd'hui, il nous paraît important de vérifier cette hypothèse dans un site présentant deux spécificités :

1. une longue séquence stratigraphique et des occupations dans des phases climatiques bien caractérisées par des données palynologiques, sédimentologiques et paléontologiques ${ }^{12}$;

2. un environnement immédiat ou proche dans lequel des ressources minérales nombreuses, diversifiées, facilement identifiables, proviennent de plusieurs types de gites ${ }^{13}$.

Le site retenu pour ce travail est Combe-Grenal.

Les changements observés dans l'approvisionnement en matières premières lithiques à la charnière OS2/OS1 d'abord au Roc Allan tous implantés dans des vallées secondaires a permis de constater que dans ce contexte l'amélioration climatique a impliqué un changement dans l'accessibilité aux gîtes: les alluvions et les dépôts de pente carbonatés ne sont plus accessibles lors du réchauffement. Il convient de voir si fréquentés lors de changements climatiques de même ordre mais plus anciens (charnière $\mathrm{OS6} / \mathrm{OS} 5$ ou peut être $\mathrm{OS4} / \mathrm{OS} 3$ ), les mêmes phénomènes se répètent. L'ensemble des sites du Pech de l'Azé devrait permettre d'entreprendre ce travail.

Enfin, il conviendra dans le futur d'aller plus loin que la simple identification de l'origine des matériaux en essayant d'estimer le nombre de blocs utilisés dans le site. Ceci est possible pour les silex des altérites où les blocs ont très souvent des caractéristiques colorimétriques spécifiques (silex du Bergeracois notamment). Cette démarche permettra de dépasser l'approche globalisante actuelle. La signification n'est pas la même si dix pièces en silex exogène sont issues d'un même bloc ou de dix blocs différents ou si cinq éclats de retouche proviennent du même bloc que le seul racloir 
présent. Ainsi une nouvelle étape sera franchie dans la compréhension du comportement humain qui reste la finalité de nos recherches.

\section{BIBLIOGRAPHIE}

ASTRUC J.-G. 1988 - Carte géologique Cahors (1/50 000) et notice, feuille 881. Orléans, B.R.G.M., 1986, 29 p., ill.

ASTRUC J.-G. 1990 - Carte géologique Gourdon (1/50 000) et notice, feuille 832.-Orléans, B.R.G.M., 1990, 45 p., ill.

BLET M. 1999 - L'apport de l'ablation laser couplée à l'ICP-MS à la caractérisation des archéomatériaux siliceux. Thèse de Doctorat de l'Université d'Orléans, dactylographiée, 217 p., 95 fig.

BONGNI F. 1994 - Contribution à l'étude de l'Aurignacien de l'Abri Pataud (Les Eyzies-de-Tayac, Dordogne). L'industrie lithique de la couche 13. Mémoire de D.E.A.. Muséum d'Histoire naturelle. I.P.H.. Paris., 1994, 74 p., 10 fig., 19 tabl. (inédit).

BONVIN-BORER P., MASSEREY C. 1981 - Etude préliminaire à la pétroarchéologie des roches siliceuses. Origine des silex de deux stations lacustres lémaniques Corsier-Port et Morges la Poudrière. Travail de diplôme en archéologie préhistorique, Département d'anthropologie et d'écologie de l'Université de Genève, dactylographié, $38 \mathrm{p}$.

BORDES F. 1972 - A tail of two caves. New-York : Harper \& Row publisher, 169 p., ill.

BORDES F., SONNEVILLE-BORDES D. (de), 1954 - Présence probable de jaspe de Fontmaure dans l'Aurignacien V de Laugerie-Haute. Bulletin de la Société Préhistorique Française t. L I, nº 1-2, 1954, p. 67-68.

BOULE M. 1892 - Note sur le remplissage des cavernes. L'Anthropologie, III, p. 21-24

BRESSY C. 1998 - Analyse géochimique et provenance du silex, une approche de trois questions sur l'origine du silex dans les sites paléolithiques à néolithiques de Chartreuses et du Vercors (France). Mémoire de D.E.A, Université Aix-Marseille I, 112 p. ill.

BRESSY C. 2002 - Caractérisation et gestion du silex des sites mésolithiques du nord-est de l'arc alpin. Une approche pétrographique et géochimique. Thèse de doctorat de l'Université d'Aix -Marseille I, 686 p.

BRESSY C., BINTZ P., POUPEAU G., BARRAT J.A. et KELLER F. 1998 - La caractérisation géochimique des sources de silex dans le massif du Vercors (France). Approche préliminaire. Cahiers d'archéologie Romande, 8 p., 3 fig.

BRESSY C., BINTZ P., POUPEAU G., BARRAT J.A., KELLER F. 1999 - ICP-MS Flint characterization and artefacts sourcing. The La Grande-Rivoire Meso- to Neolithic site case (Northren Alps, France). In : 6th International conference of non-destructive testing and microanalysis for the diagnostics and conservation of the cultural and environmental heritage. Rome, May 17th - 20th 1999.

BRICKER H.-M. 1975 - Provenience of flint used for the manufacture of tools at the Abri Pataud, les Eyzies (Dordogne). In : excavation of the Abri Pataud, les Eyzies, Dordogne, H.-L. Movius general ed. 
Cambridge, Mass.: peabody Museum, Harvard University, 1975, p. 194-197 (American School of Prehistoric Research Bulletin; 30).

CELERIER G. 1993 - L'abri sous-roche de Pont d'Ambon à Bourdeilles (Dordogne). 1 : Technologie de l'outillage taillé ; 2 : Inventaire et typomètrie des pointes aziliennes. Ed. du C.N.R/S., Gallia Préhistoire, t. 35, p. 1-98, ill.

CELERIER G. 1998 - L'abri sous-roche de Pont d'Ambon à Bourdeilles (Dordogne, France) perspective synthétique. Paléo $n^{\circ} 10$, p. 233-264.

CHADELLE J.P. 1983 - Technologie et utilisation du silex au Périgordien supérieur: l'exemple de la couche VII du Flageolet I. Toulouse, Ecole des Hautes Etudes en Sciences Sociales, 1983, 151 p., ill., (mémoire). CHALARD P., TURQ A. 2001 - Caractérisation des silex. In JARRY M., BERTRAND F., CHALARD P., COLONGE D., DEBENHAM N., DIOT M.F., LELOUVIER L.A., MOURRE V., TIXIER C., TURQ A., 2001. Les Bosses 1 ; Un gisement Paléolithique moyen antérieur à l'avant-dernier interglaciaire sur la moyenne terrasse du Lot. D.F.S. Toulouse 2001, 249 p., ill.

CHIOTTI L., LEOZ L., NESPOULET R. et POTTIER C. 2003 - Quelques exemples de stratégies d'approvisionnement dans l'Aurignacien et le Gravettien à l'abri Pataud (Dordogne). Préhistoire du Sud-ouest 2003 supplément $n^{\circ} 5$, Actes de la table ronde internationale d'Aurillac (Cantal), p. $115-123$.

COMBES J.-L. 1888 - Les mondes disparus, géologie, paléontologie et ancienneté de l'homme dans le département de Lot-et-Garonne. Agen, Imprimerie V. Lentheric, 1888, 185 p., 7 pl.

CONSIGNY S. 1996 - Origine des silex et géochimie : premiers résultats archéologiques. Cahiers archéologiques de Bourgogne. Paléolithique supérieur et Epipaléolithique dans le Nord-Est de la France. Actes de la table ronde de Dijon, 7 et 8 octobre 1995.

COULONGES L. 1935 - Les gisements préhistoriques de Sauveterre-la-Lémance (Lot-et-Garonne).- Paris, Masson, 1935, 56 p., 24 fig., 6 pl.ht., (Archives de l'Institut de Paléontologie humaine).

COULONGES L. 1963 - Magdalénien et Périgordien post-glaciaires : la grotte de La Borie del Rey (Lotet-Garonne). Gallia-Préhistoire, t. VI, 1963, p. 1-29, 16 fig., 1 tabl.

COULONGES L., SONNEVILLE-BORDES, D. (de) 1953 - Le Paléolithique du Plateau Cabrol à Saint-Frontsur-Lémance (Lot-et-Garonne). Bulletin de la Société Préhistorique Française, t. 50, fasc. 5-6, 1953, p. 333-337, 1 fig., 1 tabl.

DEMARS P.-Y. 1974 - Origine des roches utilisées dans la fabrication d'outils dans le bassin de Brive au Paléolithique Supérieur (premiers résultats). Bulletin de la Société Scientifique et Archéologique de la Corrèze, t. 96, p. 29-39, 2 fig., 1 tabl.

DEMARS P.-Y. 1980 - Les matières premières siliceuses utilisées au Paléolithique supérieur dans le bassin de Brive. Bordeaux, université de Bordeaux I, 1980, 173 p., 26 fig., (Thèse $3^{\mathrm{e}}$ cycle : Géologie du Quaternaire et préhistoire: Bordeaux I: 1980 ; 1566).

DEMARS P.-Y 1982a - L'utilisation du silex au Paléolithique supérieur : choix, approvisionnement, circulation. L'exemple du Bassin de Brive. Paris : CNRS, 1982, 253 p., fig. (Cahiers du Quaternaire, 5) DEMARS P.-Y 1982b - Origine proche ou origine lointaine des silex au Paléolithique supérieur. Une réponse à Annie Masson. Bulletin de la Société Préhistorique Française, t. 79, n 9, 1982, p. 266-267.

DEMARS P.-Y. 1990 - L'économie du silex à Laugerie-Haute (Dordogne). Cahiers du Quaternaire, $\mathrm{n}^{\circ}$ 17. Le silex de sa genèse à l'outil. Actes du V $\mathrm{V}^{\mathrm{e}}$ Colloque international sur le silex, p. 373-383, 6 fig. 
DEMARS P.Y. 1994 - L'économie du silex au Paléolithique supérieur dans le Nord de l'Aquitaine. Bordeaux : université de Bordeaux I. 2 vol., 549 p. d'analyse, 270 p. de synthèse, Thèse d'Etat : Bordeaux I : 1985, 988.

DIBBLE H.L., MCPHERRON S.P. 2001 - Pech de l'Azé IV (Carsac, Dordogne), rapport d'opération de l'année 2001. rapport de fouilles inédit, $57 \mathrm{p}$.

DUCHADEAU-KERVAZO C. 1982 - Recherche sur l'occupation paléolithique dans le Bassin de la Dronne. Bordeaux : Université de Bordeaux I, 1982, 2 t., 885 et 289 p. ill. (Thèse $3^{\mathrm{e}}$ cycle : Géologie du Quaternaire et Préhistoire : Bordeaux I : 1982 : 1755).

DUNHAM R.J. 1962 - Classification of carbonates rocks according to depositional texture. In : WE. Ham. (Ed.) Classification of carbonates rocks, Am. Assoc. Petrl. Geol., Mem. 1, p. 208-221.

FITTE P. 1970 - A propos d'un biface Moustérien de Tradition Acheuléenne en silex pressignien, découvert à Saint-Jean-Ligoure (Haute Vienne). Bulletin de la Société archéologique et Historique du Limousin, 1970, t. 97, p. 3-6.

GENESTE J.-M. 1985 - Analyse lithique d'industries moustériennes du Périgord : une approche technologique du comportement des groupes humains au Paléolithique moyen. Bordeaux : université de Bordeaux I. 2 vol., X. 572 p., 230 p. de pl., Thèse N. D. : Sc. : Bordeaux I : 1985, 2.

GREGOIRE S. 2000 - Origine des matières premières des industries lithiques du Paléolithique pyrénéen et méditerranéen. Contributions à la connaissance des aires de circulations humaines. Université de Perpignan, 1 vol., 246 p., ill. Thèse N.D. 2000.

GREGOIRE S. 2001- Apport et limites des nouvelles techniques de la pétroarchéologie préhistorique. Compte Rendus à l'Académie des Sciences. Paris, Sciences de la terre et des Planètes 332 (2001), p. 479-482.

HORAN L.J. 1977 - Preliminary results : « Fingerprinting » of archaeological flint sources, Dordogne valley, Southwestern France.Santa Cruz : University of California, 1977.

JARRY M., BERTRAND F., CHALARD P., COLONGE D., DEBENHAM N., DIOT M.F., LELOUVIER L.A., MOURRE V., TIXIER C., TURQ A., 2001 - Les Bosses 1 ; Un gisement Paléolithique moyen antérieur à l'avantdernier interglaciaire sur la moyenne terrasse du Lot. D.F.S. Toulouse 2001, 249 p., ill.

KAFA J. 1988 - Un modèle d'une plateforme carbonatée le Dogger inférieur du Quercy : sédimentation diagénèse et évolution de la porosité. Université de Pau et des Pays de l'Adour, 1988. 419 p. ill.

LARRICK R.-R. 1983 - The circulation of Solutrean foliate point cherts : residential mobility in the Perigord. Binghamton : Dept. of Anthropology, 1983, ill. (Unpublished Ph. D. Dissertation).

LARTET E., CHRISTY H. 1869 - Reliquae Aquitanicae ; being contributions to Archeology and paleontology of Périgord and the adjoining provinces of southern France. London, H. Baillière Publisher.

LE BRUN-RICALENS F. 1988 - Contribution à l'étude du Paléolithique du pays des Serres du Bas-Quercy et de l'Agenais entre les vallées du Lot et de la Garonne. Mémoire de D.E.A. Université de Toulouse II Le Mirail, 452 p., 208 fig.

LE BRUN-RICALENS F., SERONIE VIVIEN M.-R. 2004 - Présence d'un silex d'origine nord-pyrénéenne (Chalosse ?) en Haut-Quercy dans l'Aurignacien du Piage (Lot, France) et implications. Paléo ${ }^{\circ} 16$, 2004, p. 129-137.

LE TENSORER J.-M. 1979 - Recherches sur le Quaternaire en Lot-et-Garonne : stratigraphie, paléoclimatologie et préhistoire paléolithique. Bordeaux : université de Bordeaux I. IV-812 p., ill. Thèse : Sc. Bordeaux I : $1979 ; 87$. 
LENOIR M., OBRY J. et SERONIE-VIVIEN M.-R. 1998 - Occurrence of allochtones flint in Upper Paleolithic site near Bordeaux. In : Ramos Millan et Bustillo : Siliceous rocks and culture, $\mathrm{VI}^{\mathrm{e}}$ International Flint Symposium, Madrid sept. 1991, p. 385-390.

LORBLANCHET M. 1964 - Quatre bifaces des environs de la Chapelle aux Saints. Bulletin de la Société Scientifique et Archéologique de la Corrèze, t. 86, p. 9-19.

LUCAS G. 2000 - Les industries lithiques du Flageolet I (Dordogne) : approche économique, technologique, fonctionnelle et analyse spatiale. Bordeaux : Université de Bordeaux I, 2 vol., 305 p., ill. Thèse N. D. : sc. : Bordeaux I : $2000 ; 2133$.

LUEDTKE B.-E. 1978 - Chert sources and trace element analysis. American anthropology, 44 (4) p. 744-756.

LUEDTKE B.-E. 1979 - The identification of sources of cherts artefacts. American anthropology, 43 (3) p. 413-423.

LUMLEY H.(de) et BRANDI R. 1969 - Choix de la matière première pour la confection des outils de l'Acheuléen trouvés dans la cabane du Lazaret. In : une cabane acheuléenne dans la grotte du Lazaret (Nice). Paris, Société Préhistorique française, 1969, p. 171-172. (Mémoire ; 7).

MALISSEN B. 1977 - Elaboration d'une fiche de recensement des gîtes potentiels de matières premières siliceuses. Bulletin de la Société Préhistorique Française, t. 74. 1977, c.r.s.m. n 7, p. 203-205

MASSON A. 1981 - Pétroarchéologie des roches siliceuses : intérêt en Préhistoire. Lyon : université Lyon I, 1981, 100 p. (Thèse $3^{\mathrm{e}}$ cycle : sciences de la Terre - Géologie des ensembles sédimentaires: Lyon I : 1981, 1035)

MASSON A. 1982 - Circulation paléolithique : une question de longueur. Bulletin de la Société Préhistorique Française, 1982, t. 79, n 7, p. 197.

MAUGER M. 1985 - Les matériaux siliceux utilisés au Paléolithique supérieur en Ile-de-France. Occupation du territoire, déplacements et approche des mouvements saisonniers. Paris, université de Paris I, 1985, 294 p., ill. (thèse $3^{\mathrm{e}}$ cycle).

MONMEJEAN E., BORDES F. et SONNEVILLE-BORDES D. (de) 1964 - Le Périgordien supérieur à burins de Noailles du Roc de Gavaudun (Lot-et-Garonne). L'Anthropologie, t. 68, n 3-4, p. 253-316, 33 fig., 4 tabl.

MONNIER J.-L. 1980 - Le Paléolithique de la Bretagne dans son cadre géologique. Rennes : université de Rennes, 1980.

MONNIER, J.-L. 1988 - Chronostratigraphie et écologie des industries paléolithiques de la Bretagne. Actes du colloque Cultures et industries paléolithiques en milieu loessique. Amines 9-11 décembre 1986. Revue archéologique de Picardie n 1-2, 1988, p. 75-80.

MORALA A. 1979 - Etude préliminaire de la station aurignacienne des Ardailloux, commune de Soturac (lot). Bulletin de la Société des études du Lot, fasc. 3, juillet septembre, p. 185-201, 6 fig., 4 tabl.

MORALA A. 1980 - Observations sur le Périgordien, l'Aurignacien et leurs matières premières lithiques en Haut-Agenais. Toulouse, Ecole des Hautes études en Sciences Sociales, 1980, 191 p., ill. (Mémoire). MORALA A. 1983 - A propos des matières premières lithiques en Haut-Agenais. Bulletin de la Société Préhistorique Française, t. 80, nº 6, 1983, p. 169. 
MORALA A. 1989 - Les voies de communication au Paléolithique supérieur en Aquitaine du Nord : l'exemple du Haut-Agenais. Paléo 1, 1989, p. 31-35, ill.

MORALA A., TURQ A. 1990 - Les stratégies d'exploitation du milieu minéral, du Riss à l'Holocène, en Haut-Agenais (Sud-ouest de la France). Cahiers du Quaternaire, $\mathrm{n}^{\circ} 17$. Le silex de sa genèse à l'outil. Actes du V $\mathrm{V}^{\mathrm{e}}$ Colloque international sur le silex, p. 405-414, 4 fig.

MORISSET C., 1996 - Caractérisation de silex archéologiques par analyse par activation protonique. Travail de fin d'études de l'Ecole Centrale de Lyon, $70 \mathrm{p}$.

OWEN W.-E., 1938 - The Kombewa Culture, Kenya colony. Man, p. 203-205.

PLATEL J.-P. 1983 - Carte géologique Fumel (1/50 000) et notice, feuille 855. Orléans, B.R.G.M., 1983, 52 p. ill.

PLATEL J.-P. 1989 - Le Crétacé de la plate-forme septentrionale du bassin d'Aquitaine, stratigraphie et évolution géodynamique. Documents du B.R.G.M. n 164, Orléans 572 p. ill.

RIGAUD J.-Ph. 1982 - Le Paléolithique en Périgord : les données du Sud-Ouest sarladais et leurs

implications. Bordeaux : université de Bordeaux I. 2 t., 497 p., fig., tabl. Thèse : Sc. : Bordeaux I :

$1982,737$.

RIO M. 1982 - Les accidents siliceux dans le crétacé du bassin vocontien (Sud-est de la France) contribution à l'étude de la silicification des formations calcaires. Docum. Laboratoire de Géologie de Lyon, $\mathrm{n}^{\circ} 84$, 1982, 178 p., 80 fig., 38 tabl., 6 pl., 1 carte ht.

RIO M., CHALAMET A. 1980 - Relations entre l'habitus, la dimension des cristallites et le comportement thermique de la silice dans divers types d'accidents siliceux. Bull. Minéralogique, vol. 103, p. 44.

SAINT-PERIER R. 1930 - La grotte d'Isturitz. I. Le Magdalénien de la Salle de Saint-Martin. Archives de l'Institut de Paléontologie Humaine, mémoire 7, 123 p., 101 fig., 13 pl.

SERONIE-VIVIEN M. 1972 - Contribution à l'étude du Sénonien en Aquitaine septentrionale. Les stratotypes: Coniacien, Santonien, Campanien. Les stratotypes français. vol. II, Paris, C.N.R.S., 1972.

SERONIE-VIVIEN M. et M.-R. 1987 - Les silex du Mésozoïque nord-aquitain : approche géologique de l'étude du silex pour servir à la recherche. Bulletin de la Société Linnéenne de Bordeaux, 1987, suppl. au t. XV, 135 p., ill.

SERONIE-VIVIEN M.-R. 1995 - La grotte de Pégourié, Caniac-du-Causse (Lot), Périgordien, Badegoulien, Azilien, Age du Bronze. Préhistoire quercinoise, supplément $\mathrm{n}^{\circ} 2,336 \mathrm{p}$.

SERONIE-VIVIEN M.-R. 2001 - La Grotte du Sanglier à Reilhac (Lot) Du Magdalénien au Néolithique ancien. Préhistoire du Sud-Ouest, supplément n 4, 2001, 182 p.

SERONIE-VIVIEN M.-R. 2003 - Origine méridionale de silex recueillis dans le Paléolithique supérieur de la région Périgord-Quercy. Préhistoire du Sud-ouest 2003 supplément n5, (actes de la table ronde internationale d'Aurillac (Cantal)) p. 305-306.

SERONIE-VIVIEN M.-R., LE TENSORER J.-M. 1977 - Données récentes sur l'Azilien du Lot : la grotte de Pégourié à Caniac du Causse. In : Colloque international du C.N.R.S., $n^{\circ} 271$, La fin des temps glaciaires en Europe-Chronostratigraphie et écologie des cultures du Paléolithique final, Bordeaux 24-28 mai 1997, p. 175-200. Prétirage.

SIMON-COINCON R., ASTRUC J.-G. 1991 - Les pièges karstiques en Quercy : rôle et signification dans l'évolution des paysages. Bulletin de la Société Géologique de France, 1991, t. 162, n 3 p. 595-605. 
SIMONNET R. 1999 - De la géologie à la Préhistoire : le silex des Prépyrénéens. Résultats et réflexions sur les perspectives et les limites de l'étude des matières premières lithiques. Paléo ${ }^{\circ}$ 11, p. 71 à 88,14 fig., 4 tabl.

SORESSI M. 2002 - Le Moustérien de tradition acheuléenne du sud-ouest de la France. Discussion sur la signification du faciès à partir de l'étude comparée de quatre sites : Pech-de-l'Azé I, Le Moustier, La Rochette et la Grotte XVI. Thèse de l'Université Bordeaux I, 330 p.

TEXIER J.-P. 1982 - Les formations superficielles du bassin de l'Isle. Paris : C.N.R.S., Centre de publication de Bordeaux. 316 p., ill., cartes h.t. Cahiers du Quaternaire ; 4.

THIEBAUT C. 2003 - L'industrie lithique de la couche III du Roc de Marsal : le problème de l'attribution d'une série lithique au Moustérien à denticulés. Paléo nº 15, p. 141-168 ill.

TORTI C. 1983 - Contribution à l'étude paléogéographique du Massif central au Paléolithique moyen et supérieur. Bulletin de la Société Préhistorique Française, t. 80, 1983, n 10-12 p. 300-307, 2 fig., 7 tabl.

TURQ A. 1977a - Le complexe d'habitat préhistorique du Plateau Cabrol. Bulletin de la Société Préhistorique Française, t. 74, Etudes et Travaux, fasc. 2, p. 489-504, 12 fig., 1 tabl.

TURQ A. 1977b - Première approche sur le Paléolithique moyen du gisement des Ardailloux, commune de Soturac (Lot). Bulletin de la Société des Etudes du Lot, t. XCVIII, fasc. 4, p. 222-242, 9 fig., 1 tabl.

TURQ A. 1987-1988 - Sauveterre-la-Lémance : Le Roc Allan. In : Rigaud J.-Ph. (dir.) Aquitaine. Galliainformations Préhistoire et Histoire, 1, p. 146-147.

TURQ A. 1988a - Le Paléolithique inférieur et moyen en Haut-Agenais : Etat des recherches. Revue de l'Agenais, $115^{\mathrm{e}}$ année, fasc. 1, p. 83-112, 7 fig., 1 tabl.

TURQ A. 1988c - Origine des roches siliceuses. In : DUBREUILH J. et al. Notice explicative de la feuille Belvès à 1/50 000. Orléans : éd. du B.R.G.M., p. 29-34.

TURQ A. 1989 - Exploitation des matières premières lithiques et exploitation du sol : l'exemple du Moustérien entre Dordogne et Lot. In : INQUA, Colloque du comité français de l'Union internationale pour l'étude du Quaternaire: Variations des paléo-milieux et peuplement préhistorique. Textes réunis par H. Laville, Paris : C.N.R.S. Centre régional de publication de Bordeaux, p.179-204, ill. Cahiers du Quaternaire ; 13.

TURQ A. 1992 - Le Paléolithique inférieur et moyen entre les vallées de la Dordogne et du Lot. Bordeaux : Université de Bordeaux I, 2 vol., 782 p., ill. Thèse N. D. : sc. : Bordeaux I : $1992 ; 778$.

TURQ A. 1999 - Caractérisation de matières premières lithiques et implications : quelques réflexions. In : séminaire du Centre d'Anthropologie organisée par F. Briois et V. Darras. La Pierre taillée : ressources, technologies, diffusion. Edition des archives d'Ecologie Préhistorique, Ecole des Hautes Etudes en Sciences Sociales. Toulouse, p. 7-13, ill.

TURQ A., 2003 - De la matière première lithique brute à la mise au jour de l'objet archéologique : propositions pour une meilleure exploitation du potentiel informatif du matériel lithique illustrées par quelques exemples du Paléolithique aquitain. Perpignan : Université de Perpignan, mémoire original pour l'obtention d'une habilitation à diriger des recherches, 165 p., CV et travaux (3 vol.).

TURQ A, MAZIERE G., KERVAZO B., DETRAIN L. et BARBIER P. 1996 - De la fin du Paléolithique supérieur à l'Epipaléolithique:Mésolithique en Haut-Agenais.In S.P.F. XXIII ${ }^{e}$ Congrès Préhistorique de France. La vie aux temps préhistoriques. Paris du 3 au 7 novembre 1989. Paris : S.P.F., 1989, p. 300-303, fig. 
TURQ A., ANTIGNAC G., ROUSSEL P. 1999 - Les silicifications coniaciennes du Sarladais et du Gourdonnais : inventaire et implications archéologiques. Paléo 11, 1999, p. 145-160.

TURQ A., DETRAIN L., VIGIER S. 2000 - L'importance de l'accessibilité des gîtes dans l'étude de l'approvisionnement en matières premières lithiques : l'exemple du Haut-Agenais (Lot-etGaronne). In : Les derniers chasseurs-cueilleurs d'Europe occidentale, Actes du colloque international de Besançon, octobre 1998. Besançon, Presses Universitaires Franc-Comtoises : (Annales Littéraires, 699 ; Série « Environnement, sociétés et archéologie », 1), p. 267-276.

VALENSI L. 1960 - De l'origine des silex protomagdaléniens de l'abri Pataud, les Eyzies. Bulletin de la Société Préhistorique Française, t. 56, n 1-2, p. 80-84.

\section{NOTES}

1. Nous regrettons que les discussions et échanges fructueux sur la terminologie qui ont eu lieu lors de la réunion des Eyzies les 6 et 7 juillet 1982 et à laquelle participaient G. Célerier, J.-P. Chadelle, P.-Y. Demars, J.-M. Geneste, R. Larrick, A. Morala, J.-Ph. Rigaud, M.-R. Séronie-Vivien, R. Séronie-Vivien, J. Tixier et nous-mêmes n'aient jamais été publiés dans leur intégralité.

2. Un bilan similaire sur l'interprétation des résultats et le commentaire des faits archéologiques sont en cours de préparation.

3. Notons toutefois qu'un examen en lumière rasante permet de déceler une série d'ondulations très nettes non observables sur d'autres matériaux.

4. On bénéficie de plus d'un siècle de cartes géologiques et de nombreuses synthèses sur les formations carbonatées (Séronie-Vivien M. 1972 ; Platel 1989).

5. Une exception, la Bretagne où les cordons littoraux ont fait l'objet d'études détaillées (Monnier 1980 et 1988).

6. Les recherches menées sur la vallée de la Vézère ont permis de mettre en évidence le même phénomène: présence de silex crétacés en amont des affleurements actuels. Une étude géomorphologique régionale serait utile pour savoir si ce phénomène est local ou plus vraisemblablement présent dans tout le Bassin aquitain.

7. Ce que semblent confirmer les rares essais sur les échantillons récoltés.

8. Sur les 23 lieux de prélèvements, les silex gris et noirs dominent 11 fois, les blonds 10 fois et dans trois cas ils sont à égalité. Des prélèvements effectués trois années de suite sur quatre sites ont montré pour trois d'entre eux des inversions de pourcentage.

9. N'oublions pas que la dimension des blocs retrouvés dans les formations dépend d'une part, des dimensions des nodules dans l'environnement et d'autre part, de la compétence de la rivière. Aujourd'hui celle-ci est bien moindre qu'en période glaciaire.

10. Ce type d'approche a été fait dès les années 1980 par J.-P. Raynal.

11. Voir figure 2

12. Rappelons que ce n'est pas le résultat d'une seule approche qui doit être pris en compte mais l'ensemble des données.

13. Tous les sites ne sont pas sur un même pied d'égalité devant l'étude des matières premières lithiques. Les gisements implantés dans des zones où les matériaux disponibles sont peu caractéristiques et proviennent du même gîte ou de gîtes proches de grandes zones, comme par exemple le Périgord avec ses silex blonds et gris noirs du Sénonien, sont peu favorables à l'analyse lithologique. 


\section{RÉSUMÉS}

Vingt cinq ans d'expérience en études lithologiques nous ont paru suffisants pour mener à bien une réflexion sur la caractérisation des matières premières lithiques et l'identification de leurs origines. Dans ce travail, sont abordés la prospection et l'inventaire des gîtes, la cartographie, les méthodes d'identification. L'analyse critique des données nous permet de mettre en évidence les principales difficultés rencontrées et ainsi de fixer les limites des recherches menées. Pour les domaines qui nous ont semblé être essentiels, des propositions concrètes sont faites pour tenter de dépasser les points de blocage. Pour améliorer nos connaissances des ressources actuelles, notamment celles des alluvions (l'une des sources les plus fréquemment utilisées par les hommes préhistoriques mais parmi les plus mal connues), une approche est proposée et des résultats présentés. Cette méthode prend en compte la morphologie et la qualité des matériaux. L'accent est également mis sur le problème majeur qui est le transfert des données du référentiel actualiste (lithothèque) au matériel archéologique : comment, à partir des ressources actuelles, peut-on envisager celles dont disposaient le ou les fabricants des objets archéologiques étudiés. Quelques exemples archéologiques servent à aborder les problèmes d'accessibilité aux gîtes et à indiquer quelques axes de recherche.

Twenty-five years of experience in lithologic studies seem sufficient to be able to characterize lithic raw material and to identify their origins. In this study, prospection and inventory of the deposits, their location and their description are considered. The critical analysis of data allows to underline the main difficulties and also to set up the limits of the present research concerning the main topics. In essential fields, concrete suggestions are made to improve our knowledge about ressources and especially alluvial deposits (one of the most frequent lithic supply for prehistorical men but also the less known). A method with its applications is presented, taking into account the morphology and the quality of lithic raw material. A major problem is introduced: the transfer of the related data (lithotheque) to the archaeological material. How can we know from today's ressources what were the real ones available for the prehistoric toolmaker knapper? Some archeological examples are used to approach the questions of deposits accessibility and to give some research issues.

\section{INDEX}

Keywords : accessibility, flint, flint sites, knapping ability, material origin

Mots-clés : accessibilité, aptitude à la taille, gîte à silex, origines des matériaux, silex

\section{AUTEUR}

\section{ALAIN TURQ}

Musée national de Préhistoire, 24620 les Eyzies de Tayac (Dordogne), UMR PACEA ( $n^{\circ}$ 5199) du CNRS. Université Bordeaux I, Avenue des Facultés, 33405 Talence Cedex. 